\title{
Cryptanalysis of 48-step RIPEMD-160
}

\author{
Gaoli Wang ${ }^{1}$, Yanzhao Shen ${ }^{2}$ and Fukang Liu ${ }^{1,3}$ \\ ${ }^{1}$ Shanghai Key Laboratory of Trustworthy Computing, School of Computer Science and Software \\ Engineering, East China Normal University, Shanghai 200062, China \\ glwangesei.ecnu.edu.cn \\ ${ }^{2}$ Key Laboratory of Cryptologic Technology and Information Security, Ministry of Education, \\ Shandong University, Jinan 250100, China \\ $63726578 @$ qq. com \\ ${ }^{3}$ School of Computer Science and Technology, Donghua University, Shanghai 201620, China \\ $1152049805 @$ @q. com
}

\begin{abstract}
In this paper, we show how to theoretically compute the step differential probability of RIPEMD-160 under the condition that only one internal variable contains difference and the difference is a power of 2 . Inspired by the way of computing the differential probability, we can do message modification such that a step differential hold with probability 1 . Moreover, we propose a semi-free-start collision attack on 48-step RIPEMD-160, which improves the best semi-free start collision by 6 rounds. This is mainly due to that some bits of the chaining variable in the $i$-th step can be computed by adding some conditions in advance, even though some chaining variables before step $i$ are unknown. Therefore, the uncontrolled probability of the differential path is increased and the number of the needed starting points is decreased. Then a semi-free-start collision attack on 48-step RIPEMD-160 can be obtained based on the differential path constructed by Mendel et al. at ASIACRYPT 2013. The experiments confirm our reasoning and complexity analysis.
\end{abstract}

Keywords: Hash functions · RIPEMD-160 - Semi-free-start collision · Generalized message modification

\section{Introduction}

Cryptographic hash functions play an important role in modern cryptography, which can be used in digital signature schemes, message authentication codes, password authentication schemes and so on. A cryptographic hash function $H$ should fulfill some security requirements, among which collision resistance is one of the key security properties. Collision resistance means it is impossible to find two distinct messages $M$ and $M^{\prime}$ such that $H(I V, M)=H\left(I V, M^{\prime}\right)$ in less than $2^{\frac{n}{2}}$ hash computations, where $n$ is the size of the hash value and $I V$ is the standard initial value of $H$. Semi-free-start collision resistance means it is impossible to find $\left(I V^{\prime}, M\right)$ and $\left(I V^{\prime}, M^{\prime}\right)$ such that $H\left(I V^{\prime}, M\right)=H\left(I V^{\prime}, M^{\prime}\right)$ in less than $2^{\frac{n}{2}}$ hash computations, where $I V^{\prime}$ is not always equal to $I V$. In Merkle-Damgård [Dam89, Mer89] construction, if the compression function is collision resistant, we can get that the corresponding hash function is also collision resistant. Thus, the semi-free-start collision attack on Merkle-Damgård hash functions is of great significance.

Because of the break-through progresses in MD-SHA hash function cryptanalysis $\left[\mathrm{WLF}^{+} 05\right.$, WY05, WYY05b, WYY05a, YWYP06, YWZW05], especially the analysis of MD5 [WY05] and SHA-1 [WYY05a], NIST started a four-year hash function competition to design a new hash standard SHA-3 [SHA]. Many techniques such as advanced message modification [WLF $\left.{ }^{+} 05\right]$ and tunnels [Kli06] are proposed in the past decade. Recently, the free-start collision attack for the full SHA-1 [SKP16] speeds up the removal of SHA-1. The SHA-2 and SHA-3 hash functions are recommended to be deployed sooner in many products and services that used to 
rely on SHA-1. Among the MD-SHA family, only SHA-2 [DEM14, MNS11, MNS13] and RIPEMD-160 [MNSS12, MPS ${ }^{+}$13] compression functions are still unbroken.

RIPEMD-family is a subfamily of the MD-SHA-family. The first hash function of the RIPEMDfamily is RIPEMD (denoted by RIPEMD-0) [BP95] which is devised in the framework of the EU project RIPE (RACE Integrity Primitives Evaluation, 1988-1992). The compression function of RIPEMD-0 consists of two parallel MD4-like functions with 48 steps. The early cryptanalysis of RIPEMD-0 was from Dobbertin [Dob97] and the practical collision attack was proposed by Wang et al. [WLF ${ }^{+}$05]. Due to the experience of evaluating MD4 and RIPEMD-0, RIPEMD-128 and RIPEMD-160 [DBP96] were proposed in 1996 by Dobbertin et al. as reinforced hash functions for RIPEMD-0. RIPEMD-128 and RIPEMD-160 were standardized by ISO/IEC [Int11], which have 128/160-bit output and 64/80 steps respectively. A series of collision attacks on reduced RIPEMD-128 are presented in [MPRR06, MNS12, WW08, Wan14, WY15] and a semi-free-start collision attack on the full RIPEMD-128 is proposed in [LP13] (the extended version is [LP16]). However, RIPEMD-160 is unbroken until now. Therefore, the continuous analysis of the security margin of RIPEMD-160 is of great significance.

\section{Related Work}

At ISC 2006, Mendel et al. [MPRR06] examined to what extent the attacks [Dob97, WLF ${ }^{+}$05] can apply to RIPEMD-128 and RIPEMD-160. At ISC 2012, Mendel et al. [MNSS12] presented a practical semi-free-start collision attack on 36-step RIPEMD-160 (not starting from the first step). Later at ASIACRYPT 2013, Mendel et al. [MPS ${ }^{+13}$ ] proposed semi-free-start collision attacks on 42-step RIPEMD-160 (not starting from the first step) and the first 36-step RIPEMD-160. As for the preimage attack, at INSCRYPT 2010, Ohtahara et al. [OSS10] gave a preimage attacks on the first 30-step and the last 31-step RIPEMD-160. At ISC 2014, Wang and Shen [WS14] presented a preimage attack on the first 34-step RIPEMD-160. Finally, at ACNS 2012, Sasaki and Wang [SW12] presented distinguishing attacks on up to 51-step RIPEMD-160 (not starting from the first step). The above are all the previous results that we are aware of on the analysis of RIPEMD-160.

In the semi-free-start collision attack on 42-step RIPEMD-160 [MPS ${ }^{+} 13$ ], the authors firstly construct relatively sparse differential paths by choosing a proper message difference. The nonlinear differential paths are constructed using very efficient automated search techniques [MNS11, MNS13]. The automated search algorithms are very effective to obtain the non-linear differential paths, and other non-linear differential path automated search algorithms include [DCR06] etc. After the differential paths in each branch of RIPEMD-160 are constructed, the authors leverage the method for using the freedom degrees proposed by Landelle and Peyrin [LP13] to get a semifree-start collision. The method [LP13] uses some message words to ensure the nonlinear parts located in the middle of both branches hold, and use the remaining message words to merge both branches. Mendel et al. $\left[\mathrm{MPS}^{+} 13\right.$ ] point out that it is hard to calculate the differential probability for each step of a given differential path of RIPEMD-160, not as the case for RIPEMD-128. This is due to the step function in RIPEMD-160 is no longer a $T$-function (a function for which the $i$-th output bit depends only on the $i$ first lower bits of all input words). The authors [MPS ${ }^{+} 13$ ] left the problem of theoretically calculating the step differential probability of RIPEMD-160 as an open problem.

\section{Our Contributions}

As a first contribution, we propose a method to theoretically calculate the real step differential probability when only one internal variable has difference and the difference is a power of 2 . That means we partially answer the open problem raised in $\left[\mathrm{MPS}^{+} 13\right]$. Furthermore, from the way of computing the differential probability, we can implement the message modification such that a step differential of RIPEMD-160 hold with probability 1. As a second and main contribution, 
Table 1: Summary of the Attacks on RIPEMD-160

\begin{tabular}{|c|c|c|c|c|}
\hline Attack Type & Steps & Generic & Complexity & Reference \\
\hline semi-free-start collision & $36^{*}$ & $2^{80}$ & practical & [MNSS12] \\
\hline semi-free-start collision & $42^{*}$ & $2^{80}$ & $2^{75.5}$ & {$\left[\mathrm{MPS}^{+} 13\right]$} \\
\hline semi-free-start collision & 36 & $2^{80}$ & $2^{70.4}$ & {$\left[\mathrm{MPS}^{+} 13\right]$} \\
\hline semi-free-start collision & $48^{*}$ & $2^{80}$ & $2^{76.4}$ & Section 3.2 \\
\hline pseudo-preimage & 30 & $2^{160}$ & $2^{148}$ & [OSS10] \\
\hline preimage & 30 & $2^{160}$ & $2^{155}$ & [OSS10] \\
\hline pseudo-preimage & $31^{*}$ & $2^{160}$ & $2^{148}$ & [OSS10] \\
\hline preimage & $31^{*}$ & $2^{160}$ & $2^{155}$ & [OSS10] \\
\hline pseudo-preimage & 34 & $2^{160}$ & $2^{155.81}$ & [WS14] \\
\hline preimage & 34 & $2^{160}$ & $2^{158.91}$ & [WS14] \\
\hline distinguishing & $48^{*}$ & & practical & [MNSS12] \\
\hline distinguishing & 51 & $2^{160}$ & $2^{158}$ & [SW12] \\
\hline
\end{tabular}

${ }^{*}$ The attack starts from an intermediate step.

we improve the semi-free start collision attack on reduced RIPEMD-160 from 42 steps to 48 steps by using the 48 -step differential path in $\left[\mathrm{MPS}^{+} 13\right]$. The improvement is mainly due to the generalized message modification technique, using which we can calculate some bits of the chaining variables $X_{37}$ and $X_{38}\left(Y_{30}\right.$ and $\left.Y_{32}\right)$ even though $X_{36}\left(Y_{29}\right.$ and $\left.Y_{31}\right)$ is unknown by adding some conditions in advance. This method was used by Landelle, Peyrin [LP13] and Mendel et al. $\left[\mathrm{MPS}^{+} 13\right]$ to improve the uncontrolled probability respectively. We extend it by incorporating more sophisticated techniques. Furthermore, some conditions of $X_{37}, X_{38}, Y_{30}$ and $Y_{32}$ can be satisfied using the message modification technique [ $\left.\mathrm{WLF}^{+} 05, \mathrm{WY} 05\right]$, which is very powerful to improve the probability of the differential path. Therefore, a semi-free start collision attack on 48-step RIPEMD-160 is obtained because the probability of the differential path is improved after the generalized message modification. The previous results and our results on RIPEMD-160 are summarized in Table 1.

\section{Organization of the Paper}

The rest of the paper is organized as follows: In Section 1, we describe the notations and the RIPEMD-160 algorithm. Section 2 proposes a method to theoretically calculate the differential probability of a given differential path of RIPEMD-160, and the message modification technique for RIPEMD-160. Section 3 shows the detailed description of the semi-free start collision attack on 48-step RIPEMD-160. Finally, we summarize the paper in Section 4.

\section{Preliminaries}

\subsection{Notations}

In order to describe our attack conveniently, we recall some notations $\left[\mathrm{WLF}^{+} 05\right]$ as follows.

1. $M=\left(m_{0}, m_{1}, \ldots, m_{15}\right)$ and $M^{\prime}=\left(m_{0}^{\prime}, m_{1}^{\prime}, \ldots, m_{15}^{\prime}\right)$ represent two 512-bit messages.

2. $X_{i}$ denotes the output of the $i$-th step for compressing $M$ in left branch, where $1 \leq i \leq 80$.

3. $Y_{i}$ denotes the output of the $i$-th step for compressing $M$ in right branch, where $1 \leq i \leq 80$.

4. $X_{i}^{\prime}$ denotes the output of the $i$-th step for compressing $M^{\prime}$ in left branch, where $1 \leq i \leq 80$.

5. $Y_{i}^{\prime}$ denotes the output of the $i$-th step for compressing $M^{\prime}$ in right branch, where $1 \leq i \leq 80$. 
6. + denotes addition modulo $2^{32}$.

7. $s$ represents the circular shift $s$ bit positions to the left.

8. $\Delta^{+} x_{i}=x_{i}^{\prime}-x_{i}$ denotes the modular difference of two words $x_{i}$ and $x_{i}^{\prime}$.

9. $\Delta x_{i}$ denotes a bitwise signed difference of two words $x_{i}$ and $x_{i}^{\prime}$. It is noted that $\Delta x_{i}$ expresses not only modular difference but also XOR difference.

10. $x_{i, j}$ represents the $j$-th bit of $x_{i}$, starting the counting from 0 .

11. $\Delta x_{i}=[j]$ denotes $x_{i, j}=0, x_{i, j}^{\prime}=1$ and $x_{i, k}=x_{i, k}^{\prime}(0 \leq k \leq 31, k \neq j)$.

12. $\Delta x_{i}=[-j]$ denotes $x_{i, j}=1, x_{i, j}^{\prime}=0$ and $x_{i, k}=x_{i, k}^{\prime}(0 \leq k \leq 31, k \neq j)$.

13. $\Delta x_{i}=\left[ \pm j_{1}, \pm j_{2}, \ldots, \pm j_{l}\right]$ denotes the $j_{1}$-th, $j_{2}$-th, $\ldots, j_{l}$-th bits of $x_{i}$ and $x_{i}^{\prime}$ are different. The "+" sign means $x_{i, j}=0$ and $x_{i, j}^{\prime}=1$, and the "-" sign means $x_{i, j}=1$ and $x_{i, j}^{\prime}=0$.

\subsection{Description of RIPEMD-160}

The hash function RIPEMD-160 compresses any arbitrary message less than $2^{64}$ length into a hash value with length of 160 bits. The input message is padded, and then processed in 512-bit blocks in the Merkle-Damgård iterative structure. For each 512-bit message block, RIPEMD-160 compresses it into a 160-bit hash value by the compression function, which is composed of two parallel operations: left branch and right branch. Each branch contains 5 rounds, and each round contains 16 steps. The boolean functions in each round are as follows:

$$
\begin{aligned}
& F_{1}(X, Y, Z)=X \oplus Y \oplus Z, \\
& F_{2}(X, Y, Z)=(X \wedge Y) \vee(\neg X \wedge Z), \\
& F_{3}(X, Y, Z)=(X \vee \neg Y) \oplus Z, \\
& F_{4}(X, Y, Z)=(X \wedge Z) \vee(Y \wedge \neg Z), \\
& F_{5}(X, Y, Z)=X \oplus(Y \vee \neg Z) .
\end{aligned}
$$

Here $X, Y, Z$ are 32-bit words. The five boolean functions are all bitwise operations. $\neg$ represents the bitwise complement of $X . \wedge, \oplus$ and $\vee$ are bitwise AND, XOR and OR respectively.

The Message Expansion. The 512-bit input message block $M$ is divided into 16 words $m_{i}$ of 32 bits each. At step $i$, the expanded message word which will be used to update left branch and right branch are denoted by $m_{\pi^{l}(i)}$ and $m_{\pi^{r}(i)}$, where the permutations $\pi^{l}(i)$ and $\pi^{r}(i)$ can be seen in Table 2.

Initialization. The input chaining variable of the compression function is denoted by $c v=$ $\left(c v_{0}, c v_{1}, c v_{2}, c v_{3}, c v_{4}\right)$, and the standard initial value can be found in [DBP96]. The initialization process of both branches are as follows:

$$
\begin{gathered}
X_{-4}=Y_{-4}=c v_{0} \ggg 10, \quad X_{-3}=Y_{-3}=c v_{4} \ggg 10, \\
X_{-2}=Y_{-2}=c v_{3} \ggg 10, \quad X_{-1}=Y_{-1}=c v_{2}, \quad X_{0}=Y_{0}=c v_{1} .
\end{gathered}
$$

State Update Transformation. The state update transformation starts from a 160-bit input chaining variable $c v=\left(c v_{0}, c v_{1}, c v_{2}, c v_{3}, c v_{4}\right)$ and updates them in 80 steps (5 rounds, each round contains 16 steps). As depicted in Figure 1, in round $j(1 \leq j \leq 5), X_{i}$ and $Y_{i}(1 \leq i \leq 80)$ are updated as follows:

$$
X_{i}=\left(X_{i-4} \lll 10\right)+\left(\left(X_{i-5} \lll 10\right)+F_{j}\left(X_{i-1}, X_{i-2},\left(X_{i-3} \lll 10\right)\right)+m_{\pi^{l}(i)}+k_{j}^{l}\right) \lll s_{i}^{l},
$$


Table 2: Order of the Message Words and Rotation Values in RIPEMD-160

\begin{tabular}{c|cccccccccccccccc}
\hline$i$ & 1 & 2 & 3 & 4 & 5 & 6 & 7 & 8 & 9 & 10 & 11 & 12 & 13 & 14 & 15 & 16 \\
\hline$\pi^{l}(i)$ & 0 & 1 & 2 & 3 & 4 & 5 & 6 & 7 & 8 & 9 & 10 & 11 & 12 & 13 & 14 & 15 \\
$\pi^{r}(i)$ & 5 & 14 & 7 & 0 & 9 & 2 & 11 & 4 & 13 & 6 & 15 & 8 & 1 & 10 & 3 & 12 \\
$s_{i}^{l}$ & 11 & 14 & 15 & 12 & 5 & 8 & 7 & 9 & 11 & 13 & 14 & 15 & 6 & 7 & 9 & 8 \\
$s_{i}^{r}$ & 8 & 9 & 9 & 11 & 13 & 15 & 15 & 5 & 7 & 7 & 8 & 11 & 14 & 14 & 12 & 6 \\
\hline$i$ & 17 & 18 & 19 & 20 & 21 & 22 & 23 & 24 & 25 & 26 & 27 & 28 & 29 & 30 & 31 & 32 \\
\hline$\pi^{l}(i)$ & 7 & 4 & 13 & 1 & 10 & 6 & 15 & 3 & 12 & 0 & 9 & 5 & 2 & 14 & 11 & 8 \\
$\pi^{r}(i)$ & 6 & 11 & 3 & 7 & 0 & 13 & 5 & 10 & 14 & 15 & 8 & 12 & 4 & 9 & 1 & 2 \\
$s_{i}^{l}$ & 7 & 6 & 8 & 13 & 11 & 9 & 7 & 15 & 7 & 12 & 15 & 9 & 11 & 7 & 13 & 12 \\
$s_{i}^{r}$ & 9 & 13 & 15 & 7 & 12 & 8 & 9 & 11 & 7 & 7 & 12 & 7 & 6 & 15 & 13 & 11 \\
\hline$i$ & 33 & 34 & 35 & 36 & 37 & 38 & 39 & 40 & 41 & 42 & 43 & 44 & 45 & 46 & 47 & 48 \\
\hline$\pi^{l}(i)$ & 3 & 10 & 14 & 4 & 9 & 15 & 8 & 1 & 2 & 7 & 0 & 6 & 13 & 11 & 5 & 12 \\
$\pi^{r}(i)$ & 15 & 5 & 1 & 3 & 7 & 14 & 6 & 9 & 11 & 8 & 12 & 2 & 10 & 0 & 4 & 13 \\
$s_{i}^{l}$ & 11 & 13 & 6 & 7 & 14 & 9 & 13 & 15 & 14 & 8 & 13 & 6 & 5 & 12 & 7 & 5 \\
$s_{i}^{r}$ & 9 & 7 & 15 & 11 & 8 & 6 & 6 & 14 & 12 & 13 & 5 & 14 & 13 & 13 & 7 & 5 \\
\hline$i$ & 49 & 50 & 51 & 52 & 53 & 54 & 55 & 56 & 57 & 58 & 59 & 60 & 61 & 62 & 63 & 64 \\
\hline$\pi^{l}(i)$ & 1 & 9 & 11 & 10 & 0 & 8 & 12 & 4 & 13 & 3 & 7 & 15 & 14 & 5 & 6 & 2 \\
$\pi^{r}(i)$ & 8 & 6 & 4 & 1 & 3 & 11 & 15 & 0 & 5 & 12 & 2 & 13 & 9 & 7 & 10 & 14 \\
$s_{i}^{l}$ & 11 & 12 & 14 & 15 & 14 & 15 & 9 & 8 & 9 & 14 & 5 & 6 & 8 & 6 & 5 & 12 \\
$s_{i}^{r}$ & 15 & 5 & 8 & 11 & 14 & 14 & 6 & 14 & 6 & 9 & 12 & 9 & 12 & 5 & 15 & 8 \\
\hline$i$ & 65 & 66 & 67 & 68 & 69 & 70 & 71 & 72 & 73 & 74 & 75 & 76 & 77 & 78 & 79 & 80 \\
\hline$\pi^{l}(i)$ & 4 & 0 & 5 & 9 & 7 & 12 & 2 & 10 & 14 & 1 & 3 & 8 & 11 & 6 & 15 & 13 \\
$\pi^{r}(i)$ & 12 & 15 & 10 & 4 & 1 & 5 & 8 & 7 & 6 & 2 & 13 & 14 & 0 & 3 & 9 & 11 \\
$s_{i}^{l}$ & 9 & 15 & 5 & 11 & 6 & 8 & 13 & 12 & 5 & 12 & 13 & 14 & 11 & 8 & 5 & 6 \\
$s_{i}^{r}$ & 8 & 5 & 12 & 9 & 12 & 5 & 14 & 6 & 8 & 13 & 6 & 5 & 15 & 13 & 11 & 11 \\
\hline & & & & & & & & & & & & & & &
\end{tabular}

$$
Y_{i}=\left(Y_{i-4} \lll 10\right)+\left(\left(Y_{i-5} \lll 10\right)+F_{6-j}\left(Y_{i-1}, Y_{i-2},\left(Y_{i-3} \lll 10\right)\right)+m_{\pi^{r}(i)}+k_{j}^{r}\right) \lll s_{i}^{r},
$$

where the boolean function $F_{j}$, the constants $k_{j}^{l}$ and $k_{j}^{r}$ depend on round $j\left(j=\left\lfloor\frac{i+16}{16}\right\rfloor\right)$ and left/right branch. The order of message words $\pi^{l}(i), \pi^{r}(i)$ and the details of the shift positions $s_{i}^{l}, s_{i}^{r}$ can be seen in Table 2. For the details of RIPEMD-160, we refer to [DBP96].

The Finalization. The output of compressing the block $M$ is obtained by combining the initial value $c v$ with the outputs of both branch operations. The five 32-bit words $c v_{i}^{\prime}$ composing the output chaining variable are calculated by:

$$
\begin{array}{ll}
c v_{0}^{\prime}=c v_{1}+X_{79}+\left(Y_{78} \lll 10\right), & c v_{1}^{\prime}=c v_{2}+\left(X_{78} \lll 10\right)+\left(Y_{77} \lll 10\right), \\
c v_{2}^{\prime}=c v_{3}+\left(X_{77} \lll 10\right)+\left(Y_{76} \lll 10\right), & c v_{3}^{\prime}=c v_{4}+\left(X_{76} \lll 10\right)+Y_{80}, \\
c v_{4}^{\prime}=c v_{0}+X_{80}+Y_{79} . &
\end{array}
$$

\section{Some Properties and the Message Modification}

For RIPEMD-160, Mendel et al. [MPS ${ }^{+} 13$ ] pointed out that it is difficult to theoretically calculate the differential probability for each step of a given differential path and left it as an open problem. Daum [Dau05] proposed a method to calculate the probability that $X$ satisfies the equation $(X+$ $\left.C_{0}\right) \lll S=(X \lll S)+C_{1}$ (i.e., $\left.\operatorname{Pr}\left[X \mid\left(X+C_{0}\right) \lll S=(X \lll S)+C_{1}\right]\right)$, where $C_{0}$ and $C_{1}$ are random constants. We find that from the calculation of such a probability, the probability of the modular difference of the differential probability can be calculated. Moreover, from Theorem 2 in [FLN], we can also compute the differential probability for each step under some particular 


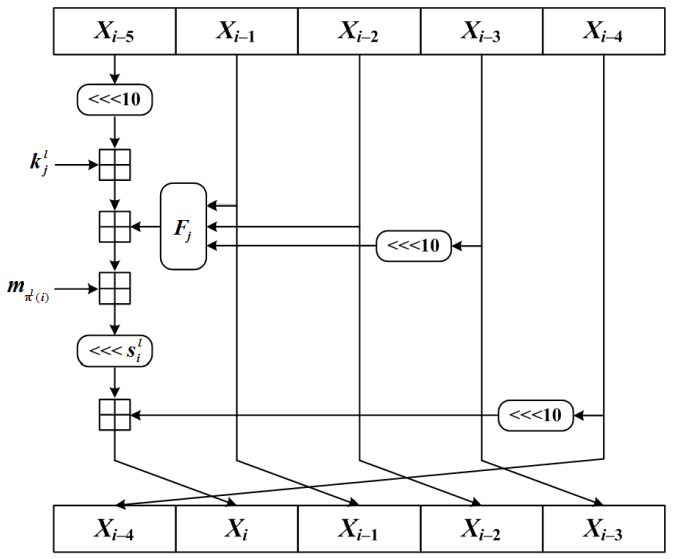

Figure 1: The left branch of step update transformation of RIPEMD-160

circumstances. In this section, we present some properties to compute the differential probability accurately under the circumstance that only one internal state word contains difference and the difference is a power of 2 . Moreover, the way of computing the differential probability sheds light on the message modification, so we propose a method to implement the message modification such that the sufficient conditions of a step differential hold with probability 1 after message modification.

\subsection{Calculating the Differential Probability}

In order to express the following propositions easily, we simplify the $i$-th step function

$$
X_{i}=\left(X_{i-4} \lll 10\right)+\left(\left(X_{i-5} \lll 10\right)+F_{j}\left(X_{i-1}, X_{i-2},\left(X_{i-3} \lll 10\right)\right)+m_{\pi^{l}(i)}+k_{j}^{l}\right) \lll s_{i}^{l}
$$

as $X_{i}=\left(X_{i-4} \lll 10\right)+\left(\left(X_{i-5} \lll 10\right)+f\left(X_{i-1}, X_{i-2},\left(X_{i-3} \lll 10\right)\right)+m+k\right) \lll s$, where $m, k$ and $s$ depend on the step $i$. If $\Delta X_{i-1}, \Delta X_{i-2}, \Delta X_{i-3}, \Delta X_{i-5}$ and $\Delta m$ are all zero, for any given value $\alpha$, the probability of $\Delta X_{i}=\alpha$ can be calculated easily as in RIPEMD-128. However, if $\Delta X_{i-5}$, $\Delta f\left(X_{i-1}, X_{i-2},\left(X_{i-3} \lll 10\right)\right)$ or $\Delta m$ is not zero, then the probability of $\Delta X_{i}=\alpha$ can not be computed as the case for RIPEMD-128. When there is only one internal state word containing difference among $X_{i-1}, X_{i-2}, X_{i-3}, X_{i-5}$ and $m$, and the difference is a power of 2, the following propositions can be used to compute the step differential probability.

Proposition 1. Let $y=x_{1}+\left(x_{2}+x_{3}\right) \lll s, y^{\prime}=x_{1}+\left(x_{2}+x_{3}^{\prime}\right) \lll s$, here $x_{1}, x_{2}, x_{3}$ and $x_{3}^{\prime}$ are 32-bit words. If $\Delta x_{3}=[i]\left(\right.$ i.e., $\left.x_{3, i}=0, x_{3, i}^{\prime}=1, x_{3, k}=x_{3, k}^{\prime}(0 \leq k \leq 31, k \neq i)\right)$, then the probability that $\Delta^{+} y$ equals to $2^{i+s(\bmod 32)}$ can be computed as follows:

$$
\operatorname{Pr}\left[\Delta^{+} y=2^{i+s(\bmod 32)}\right]=\left\{\begin{array}{cc}
1-\frac{1}{2^{32-s-i}}, & i+s \leq 31, \\
1-\frac{1}{2^{32-i}}, & i+s>31 .
\end{array}\right.
$$

Proof. Let $r_{1}=x_{2}+x_{3}, r_{2}=r_{1} \lll s, r_{1}^{\prime}=x_{2}+x_{3}^{\prime}, r_{2}^{\prime}=r_{1}^{\prime} \lll s$, then it is obvious that

$$
\Delta^{+} y=\Delta^{+} r_{2}
$$

Therefore,

$$
\operatorname{Pr}\left[\Delta^{+} y=2^{i+s(\bmod 32)}\right]=\operatorname{Pr}\left[\Delta^{+} r_{2}=2^{i+s(\bmod 32)}\right] .
$$


Case 1: When $i+s \leq 31$, then

$$
\begin{array}{rlrl}
\Delta^{+} r_{2}=2^{i+s} & \Longleftrightarrow & \Delta r_{1} & =[i] \\
& \text { or } & \Delta r_{1} & =[-i, i+1] \\
& \text { or } & \Delta r_{1} & =[-i,-(i+1), i+2] \\
& \ldots \\
& \text { or } & \Delta r_{1}=[-i,-(i+1), \ldots,-(30-s), 31-s] .
\end{array}
$$

From $\Delta x_{3}=[i]$ we can get $\Delta^{+} r_{1}=2^{i}$. Thus $\Delta r_{1}=$ [i] (i.e. $\Delta^{+} r_{1}=2^{i}$ and $r_{1, i}=0$ ) holds with probability $\frac{1}{2}$. In a similarly way, $\Delta r_{1}=[-i, i+1]$ (i.e. $\Delta^{+} r_{1}=2^{i}, r_{1, i}=1$ and $r_{1, i+1}=0$ ) holds with probability $\frac{1}{2^{2}}$. Therefore, the probability of event (1) is $\frac{1}{2}+\frac{1}{2^{2}}+\frac{1}{2^{3}}+\ldots+\frac{1}{2^{32-s-i}}=1-\left(\frac{1}{2}\right)^{32-s-i}$. Case 2: When $i+s>31$, then

$$
\begin{aligned}
& \Delta^{+} r_{2}=2^{i+s-32} \Longleftrightarrow \Delta r_{1}=[i] \\
& \text { or } \Delta r_{1}=[-i, i+1] \\
& \text { or } \Delta r_{1}=[-i,-(i+1), i+2] \\
& \text { or } \Delta r_{1}=[-i,-(i+1), \ldots,-30,31] \text {. }
\end{aligned}
$$

It is easy to see that the probability of event (2) is $\frac{1}{2}+\frac{1}{2^{2}}+\frac{1}{2^{3}}+\ldots+\frac{1}{2^{32-i}}=1-\left(\frac{1}{2}\right)^{32-i}$.

Corollary 1 Let $y=x_{1}+\left(x_{2}+x_{3}\right) \lll s, y^{\prime}=x_{1}+\left(x_{2}+x_{3}^{\prime}\right) \lll s$, here $x_{1}, x_{2}, x_{3}$ and $x_{3}^{\prime}$ are 32-bit words. If $\Delta x_{3}=[-i]$ (i.e., $\left.x_{3, i}=1, x_{3, i}^{\prime}=0, x_{3, k}=x_{3, k}^{\prime}(0 \leq k \leq 31, k \neq i)\right)$, then we can get

$$
\operatorname{Pr}\left[\Delta^{+} y=-2^{i+s(\bmod 32)}\right]=\left\{\begin{array}{cc}
1-\frac{1}{2^{32-s-i}}, & i+s \leq 31, \\
1-\frac{1}{2^{32-i}}, & i+s>31 .
\end{array}\right.
$$

\subsection{Message Modification}

In order to find a collision of a Merkle-Damgård hash function (esp. RIPEMD-160), a high probability (after the accelerating process) differential path must be constructed on the basis of a proper differences of the message and/or the initial value. Then a set of sufficient conditions that ensure the differential path hold is deduced. In order to find a pair of messages which satisfy the sufficient conditions, many accelerating techniques are proposed. Message modification [WY05, WYY05a] is one of the powerful techniques to accelerate the process of finding a pair of messages following the differential path.

For the sufficient conditions of each step of a given differential path of RIPEMD-128, it is easy to use the message modification techniques to fulfill the conditions. However, it is not the case for RIPEMD-160 due to the step function of RIPEMD-160 is not a $T$-function (i.e., the $i$-th output bit depends only on the $i$ first lower bits of all input words). The derivation process of the sufficient conditions of the differential path in RIPEMD-160 is different from that in RIPEMD-128, which will be illustrated through the following example. The differential characteristic in step 37 of the left branch is

$$
\begin{gathered}
\left(\Delta X_{32}=[-10,-16], \Delta X_{33}=[-21,-24,30], \Delta X_{34}=[-7], \Delta X_{35}=[21,24], \Delta X_{36}=[-7]\right) \\
\longrightarrow \Delta X_{37}=[21],
\end{gathered}
$$

and

$$
X_{37}=\left(X_{33} \lll 10\right)+\left(\left(X_{32} \lll 10\right)+F_{3}\left(X_{36}, X_{35},\left(X_{34} \lll 10\right)\right)+m_{9}+k_{3}^{l}\right) \lll 14 .
$$


According to the structure of the nonlinear function $F_{3}$, the conditions $X_{35,7}=1, X_{34,29}=1$, $X_{36,21}=0, X_{34,11}=1$ and $X_{36,24}=1$ ensure that $\left(\Delta X_{36}=[-7], \Delta X_{35}=[21,24], \Delta X_{34}=[-7]\right)$ result in $\Delta F_{3}\left(X_{36}, X_{35},\left(X_{34} \lll 10\right)\right)=[7,21, \pm 17]$. Denote

$$
T_{37}=\left(X_{32} \lll 10\right)+F_{3}\left(X_{36}, X_{35},\left(X_{34} \lll 10\right)\right)+m_{9}+k_{3}^{l}, \quad P_{37}=T_{37} \lll 14,
$$

obviously, we anticipate that the differences [ \pm 17$]$ in $\Delta F_{3}$ cancels out the difference [-21] in $\Delta X_{33}$. However, if the differences $[ \pm 17]$ in $\Delta F_{3}$ spreads to the 18-th bit, then the corresponding difference of $P_{37}$ not only locates in bit 31, but also in bit 0 . Thus, $\Delta^{+} X_{37}=2^{21}$ can not be hold. Therefore, the set of conditions $X_{35,7}=1, X_{34,29}=1, X_{36,21}=0, X_{34,11}=1, X_{36,24}=1$ and $X_{37,21}=0$ is not a set of sufficient conditions of the 37-th step differential path. Thus, in the message modification, we must make sure $\Delta^{+} X_{37}=2^{21}$ and $X_{37,21}=0$ hold simultaneously. So the message modification for RIPEMD-160 is very complex. We present two examples (the second example is illustrated in Appendix B) to illustrate part of the process of the message modification.

Example 1. For the $i$-th step function $X_{i}=\left(X_{i-4} \lll 10\right)+\left(\left(X_{i-5} \lll 10\right)+f\left(X_{i-1}, X_{i-2}\right.\right.$, $\left.\left.\left(X_{i-3} \lll 10\right)\right)+m+k\right) \lll s$, where $m, k$ and $s$ depend on the step $i$. Denote

$$
\begin{gathered}
r_{1}=\left(X_{i-5} \lll 10\right)+f\left(X_{i-1}, X_{i-2},\left(X_{i-3} \lll 10\right)\right)+m+k, \\
r_{1}^{\prime}=\left(X_{i-5} \lll 10\right)+f\left(X_{i-1}, X_{i-2},\left(X_{i-3} \lll 10\right)\right)+m^{\prime}+k, \\
r_{2}=r_{1} \lll s, \quad r_{2}^{\prime}=r_{1}^{\prime} \lll s .
\end{gathered}
$$

Let $\Delta^{+} m=2^{30}$ and $s=3$, in order to ensure $\Delta X_{i}=[1]$, the message modification can be processed as follows.

Step 1. If $\Delta^{+} X_{i} \neq 2$, from the proof of Proposition 1 , we know that $\Delta r_{1}=[-30,-31]$, i.e. $r_{1,30}=r_{1,31}=1$ and $r_{1,30}^{\prime}=r_{1,31}^{\prime}=0$. The message word $m$ can be modified in the following three different ways:

$$
m \longleftarrow m \pm 2^{31},
$$

then after this modification, the most two significant bits of $r_{1}$ and $r_{1}^{\prime}$ are $r_{1,30}=1, r_{1,31}=0, r_{1,30}^{\prime}=0$ and $r_{1,31}^{\prime}=1$, which means $\Delta r_{1}=[-30,31]$, thus $\Delta r_{2}=[-1,2]$. Therefore, $\Delta^{+} X_{i}=-2+2^{2}=2$. (2)

$$
m \longleftarrow m-2^{30},
$$

then after this modification, the most two significant bits of $r_{1}$ and $r_{1}^{\prime}$ are $r_{1,30}=0, r_{1,31}=1$, $r_{1,30}^{\prime}=1$ and $r_{1,31}^{\prime}=1$, which means $\Delta r_{1}=[30]$, thus $\Delta r_{2}=[1]$. Therefore, $\Delta^{+} X_{i}=2$.

$$
m \longleftarrow m+2^{30},
$$

then after this modification, the most two significant bits of $r_{1}$ and $r_{1}^{\prime}$ are $r_{1,30}=0, r_{1,31}=0$, $r_{1,30}^{\prime}=1$ and $r_{1,31}^{\prime}=0$, which means $\Delta r_{1}=[30]$, thus $\Delta r_{2}=[1]$. Therefore, $\Delta^{+} X_{i}=2$.

Step 2. If $X_{i, 1} \neq 0$, we modify the message word $m$ according to the following three circumstances.

(1) If $r_{1,30}=0$ and $r_{1,31}=0$ (i.e. $r_{1,30}^{\prime}=1$ and $r_{1,31}^{\prime}=0$ ), $m$ can be modified as: $m \longleftarrow m+2^{30}$. After the modification, the most two significant bits of $r_{1}$ and $r_{1}^{\prime}$ are $r_{1,30}=1, r_{1,31}=0, r_{1,30}^{\prime}=0$ and $r_{1,31}^{\prime}=1$. On the one hand, $r_{1,30}$ is flipped, so $r_{2,1}$ is flipped, thus $X_{i, 1}$ is flipped. On the other, after the modification, there is $\Delta^{+} r_{2}=-2+2^{2}=2$. Therefore, $\Delta^{+} X_{i}=2$ still holds.

(2) If $r_{1,30}=0$ and $r_{1,31}=1$, (i.e. $r_{1,30}^{\prime}=1$ and $r_{1,31}^{\prime}=1$ ), $m$ can be modified as: $m \longleftarrow m-2^{30}$. After the modification, the most two significant bits of $r_{1}$ and $r_{1}^{\prime}$ are $r_{1,30}=1, r_{1,31}=0, r_{1,30}^{\prime}=0$ 

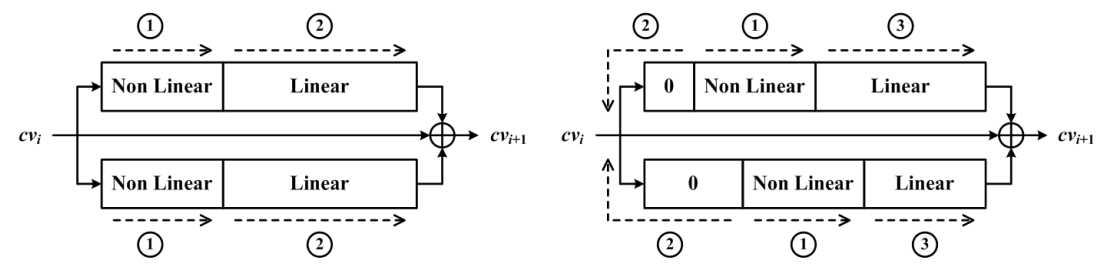

Figure 2: The traditional (left-hand side) and new (right-hand side) approach for collision search on double-branch compression functions [LP13]

and $r_{1,31}^{\prime}=1$. Therefore, $X_{i, 1}$ is flipped and $\Delta^{+} X_{i}=2$ still holds.

(3) If $r_{1,30}=1$ and $r_{1,31}=0$, (i.e. $r_{1,30}^{\prime}=0$ and $r_{1,31}^{\prime}=1$ ), $m$ can be modified as: $m \longleftarrow m \pm 2^{30}$. After the modification, $X_{i, 1}$ is flipped and $\Delta^{+} X_{i}=2$ still holds.

\section{Improved Semi-free-start Collision Attack on Reduced RIPEMD-160}

The key point of collision attacks on RIPEMD family hash functions is searching a low probability non-linear part and a high probability linear part. In the traditional collision attacks [Dob97, MNS12, MNSS12, Wan14, $\mathrm{WLF}^{+} 05$ ], the non-linear parts are located in the first steps and linear parts in the remaining ones. This restricts the space of the possible differential paths and usually fail to implement a collision attack. In this circumstance, Landelle and Peyrin [LP13] propose a new method to utilize both the freedom of the message words and the freedom of the initial value, which gives a semi-free-start collision attack on the full RIPEMD-128. The non-linear part is fixed in the intermediate steps. Some message words are used to ensure the non-linear part hold and the remaining ones are used to merge both branches. Figure 2 shows the differences between the previous strategy and the strategy in [LP13].

Mendel et al. [MPS ${ }^{+} 13$ ] constructs a differential path for 48-step RIPEMD-160, which is located between steps 17-64. Using the differential path, they obtain a semi-free-start collision attack on 42-step RIPEMD-160 with complexity $2^{75.5}$. Meanwhile, they measure that the probability of the differential path from step 58 to 64 is about $2^{-11.3}$. So the complexity of 48 -step attack is about $2^{86.8}$. Obviously, if the differential probability between steps $17-58$ is improved, the collision attack on 48-step RIPEMD-160 may be obtained.

In this section, we provide a method to compute the values of $X_{37, i}(i=2,7,17,21), X_{38, i}$ $(i=17,21), Y_{30, i}(i=9,15,21,27,30,31)$ and $Y_{32,20}$ by adding some conditions on the chaining variables in advance, even though $X_{36}, Y_{29}$ and $Y_{31}$ are unknown. Thus, the complexity of the collision attack on 42-step RIPEMD-160 is decreased and a semi-free-start collision attack on 48-step RIPEMD-160 is constructed.

\subsection{Review the General Strategy of the Attack on 42-step RIPEMD-160}

At a high level, the process of getting a semi-free-start collision attack on 42-step RIPEMD-160 $\left[\mathrm{MPS}^{+} 13\right]$ consists of the following three phases:

Phase 1: A 48-step differential path (between steps 17-64) is constructed by inserting differences in the message word $m_{7}$.

Phase 2: The message words $m_{i}(0 \leq i \leq 15, i \neq 1,4,7,13)$ are modified such that the conditions between steps 17-35 and $X_{37, i}(i=2,7)$ in the left branch and the conditions between steps 17-28 in the right branch are fulfilled. The candidate (the internal state variables and the corresponding message words) at the end of this phase is called a starting point. 
Phase 3: Using the remaining free message words $m_{1}, m_{4}, m_{7}$ and $m_{13}$ to merge the initial states of both branches to the same value. If the rest conditions of the differential path of 42-step RIPEMD-160 are fulfilled, a semi-free-start collision is obtained.

In phase 2 of searching a starting point, firstly, the internal states words $\left(X_{26}, X_{27}, X_{28}, X_{29}, X_{30}\right)$ in the left branch and $\left(Y_{21}, Y_{22}, Y_{23}, Y_{24}, Y_{25}\right)$ in the right branch are fixed. Then the message words are modified in the following order $m_{11}, m_{15}, m_{8}, m_{3}, m_{12}, m_{14}, m_{10}, m_{2}, m_{5}, m_{9}, m_{0}$ and $m_{6}$. That means the conditions in the internal states in both branches are satisfied in the following order: $X_{31}, Y_{26},\left(X_{32}, Y_{27}\right), X_{33}, Y_{28},\left(X_{25}, Y_{20}\right),\left(X_{34}, X_{35}, Y_{19}\right), X_{24},\left(X_{23}, Y_{18}\right),\left(X_{22}, X_{37,2}, X_{37,7}\right)$, $\left(X_{21}, X_{20}, X_{19}, X_{18}\right)$ and $X_{17}$. After finding a starting point, the remaining free message words $m_{1}$, $m_{4}, m_{7}$ and $m_{13}$ are used to make sure that the five initial words of both branches have a match, i.e., $X_{i}=Y_{i}(i=12,13,14,15,16)$, which holds with a probability of $2^{-32}$.

After Phase 2 and Phase 3, the sufficient conditions in steps 36-58 of the left branch (except $X_{37,2}=0$ and $X_{37,7}=1$ ) and the sufficient conditions in steps 29-58 of the right branch are not modified. [MPS ${ }^{+} 13$ ] measures the probability of both branches by experiment. The probability of the differential path in steps 36-58 of the left branch (except $X_{37,2}=0$ and $X_{37,7}=1$ ) is $2^{-8.8}$. The probability of the differential path in steps $29-58$ of the right branch is $2^{-36.6}$. Moreover, the probability of merging the initial states of both branches to the same value in Phase 3 is $2^{-32}$. Therefore, the uncontrolled probability of the differential path of 42-step RIPEMD-160 is $2^{-32} \times 2^{-8.8} \times 2^{-36.6}=2^{-77.4}$. Thus, in Phase 2 , it needs to generate $2^{77.4}$ starting points, which requires $2^{73} 42$-step RIPEMD-160. Moreover, the merging costs about $2^{-1.9}$ calls of the 42-step compression function. Therefore, the complexity of the semi-free start collision attack on 42-step RIPEMD-160 is $2^{73}+2^{77.4-1.9} \approx 2^{75.5}$.

\subsection{Semi-free-start Collision Attack on 48-step RIPEMD-160}

Mendel et al. [MPS ${ }^{+} 13$ ] proposes a differential path for 48-step RIPEMD-160 (in Table 7) and gets a collision attack on 42-step RIPEMD-160. The conditions on $X_{i}(i=36,37,38,57, \ldots, 64)$ and $Y_{i}(i=29, \ldots, 33,60, \ldots, 64)$ of the differential path $\left[\mathrm{MPS}^{+} 13\right]$ are presented in Table 8 and Table 9 . In this section, by using the generalized message modification technique, we can obtain a semi-free-start collision attack on 48-step RIPEMD-160 by decreasing the number of the starting points needed in Phase 3.

In Phase 2 of searching a starting point, firstly, similar to [MPS $\left.{ }^{+} 13\right]$, we fix the internal state variables $\left(X_{26}, X_{27}, X_{28}, X_{29}, X_{30}\right)$ in the left branch and $\left(Y_{21}, Y_{22}, Y_{23}, Y_{24}, Y_{25}\right)$ in the right branch. Then the message modification is used to make sure the conditions on the chaining variables $X_{i}(i=17, \ldots, 25,31, \ldots, 35)$ and $Y_{i}(i=18,19,20,26,27,28)$ hold. Moreover, the message words $m_{1}, m_{4}, m_{7}$ and $m_{13}$ are used to merge the initial values of both branches, i.e., $X_{i}=Y_{i}$ $(i=12,13,14,15,16)$ in Phase 3 , thus $X_{36}, Y_{29}$ and $Y_{31}$ (depend on $m_{4}, m_{4}$ and $m_{1}$ respectively) are unknown.

However, by adding some other conditions on the chaining variables in both branches in advance, we can calculate the values of $X_{37, i}(i=2,7,17,21), X_{38, i}(i=17,21)$ of the left branch and $Y_{30, i}(i=9,15,21,27,30,31), Y_{32,20}$ of the right branch correctly. The computation of the value of these bits is verified by the experiment. Furthermore, the conditions of $X_{37, i}(i=2,7,17,21)$, $X_{38, i}(i=17,21), Y_{30, i}(i=15,21,27,30,31)$ and $Y_{32,20}$ can be fulfilled by modifying or searching the message words. The above 12 conditions are marked with blue in Table 7 . That means we can ensure 10 more conditions hold in the starting point compared with [MPS $\left.{ }^{+} 13\right]$. Therefore, the probability of the differential path can be improved and the needed starting points in phase 3 is decreased after the generalized message modification. Thus, a semi-free-start-collision attack on 48-step RIPEMD-160 can be obtained. The comparison of the starting points in [MPS ${ }^{+} 13$ ] and in this paper are shown in Table 4. The procedure of the above calculation is illustrated in Table 3.

The process of computing $X_{37, i}(i=2,7,17,21), X_{38, i}(i=17,21), Y_{30, i}(i=9,15,21,27,30,31)$ and $Y_{32,20}$ is independent of $X_{36}, Y_{29}$ and $Y_{31}$ which follow the differential paths. The calculation is described in the following. Furthermore, experiments are conducted, which confirm our reasoning and calculation process. Our C implementation can be found in [ver16a, ver16b]. 
Table 3: The Process of Finding a Starting Point

\begin{tabular}{|c|c|c|c|c|c|c|}
\hline Step & 1 & 2 & 3 & 4 & 5 & 6 \\
\hline Message word & $m_{11}$ & $m_{15}$ & $m_{8}$ & $m_{3}$ & $m_{14}$ & $m_{10}$ \\
\hline MCV & $X_{31}$ & $Y_{26}$ & $X_{32}, Y_{27}$ & $X_{33}$ & $X_{25}, Y_{20}$ & $X_{34}$ \\
\hline Step & 7 & 8 & 9 & 10 & 11 & 12 \\
\hline Message word & $m_{2}$ & $m_{5}$ & $m_{12}$ & $m_{9}$ & $m_{0}$ & $m_{6}$ \\
\hline MCV & $X_{24}, X_{35}, Y_{19}$ & $X_{23}, Y_{18}$ & $Y_{28}$ & $\begin{array}{c}X_{22}, X_{37}, X_{38}, \\
Y_{30}, Y_{32}\end{array}$ & $\begin{array}{c}X_{21}, X_{20}, \\
X_{19}, X_{18}\end{array}$ & $X_{17}$ \\
\hline
\end{tabular}

MCV denotes Modified Chaining Variable.

Table 4: The Comparison of the Components of the Starting Points

\begin{tabular}{|l|r|}
\hline The Components of the Starting Points & Reference \\
\hline$X_{i}(i=17, \ldots, 35), Y_{i}(i=18, \ldots, 28), X_{37, i}(i=2,7)$ & {$\left[\mathrm{MPS}^{+} 13\right]$} \\
\hline$X_{i}(i=17, \ldots, 35), Y_{i}(i=18, \ldots, 28)$, & Section 4.2 \\
$X_{37, i}(i=2,7,17,21), X_{38, i}(i=17,21), \Delta X_{37}=0 \times 200000, \Delta X_{38}=0$ & \\
$Y_{30, i}(i=9,15,21,27,30,31), Y_{32,20}, \Delta Y_{30}=0 \times 8200000$ & \\
\hline
\end{tabular}

Calculate $X_{37, i}(i=2,7,17,21)$

The message word $m_{4}$ is used in the process of merging both branches, so in the process of finding a starting point, $X_{36}$ is unknown. From $F_{3}\left(X_{36}, X_{35},\left(X_{34} \lll 10\right)\right)=\left(X_{36} \vee \neg X_{35}\right) \oplus$ $\left(X_{34} \lll 10\right)$, we know that $X_{36}$ will have no influence on the output of the boolean function $F_{3}$ if $X_{35}=0$ is satisfied. Therefore,

$$
X_{37}=\left(X_{33} \lll 10\right)+\left(\left(X_{32} \lll 10\right)+F_{3}\left(X_{36}, X_{35},\left(X_{34} \lll 10\right)\right)+m_{9}+k_{3}^{l}\right) \lll 14
$$

can be calculated under the condition that $X_{35}=0$.

1. In order to calculate $X_{37, i}(i=17,21)$, the conditions $X_{35, i}=0(i=0, \ldots, 3,5,6)$ are added (there is the condition $X_{35,7}=1$ in the differential path). Then bits $0-3,5,6$ of $F_{3}\left(X_{36}, X_{35},\left(X_{34} \lll 10\right)\right)$ can be calculated. Let

$$
T_{1}=\left(X_{32} \lll 10\right)+\left(\left(\neg X_{34} \lll 10\right) \wedge 0 \mathrm{xef}\right)+\left(m_{9} \wedge 0 \mathrm{xef}\right)+k_{3}^{l},
$$

then $T_{1}$ can be deduced. We can choose $m_{9}$ such that $T_{1, i}=0(i=2,3,5)$. Meanwhile, we add the extra condition $X_{33, i}=0(i=6,8,9)$ which is ensured to hold by modifying $m_{3}$. Thus, the carry coming from bit 16 (19) to bit 17 (20) in the process of computing $X_{37}$ is stopped. Thanks to $X_{36,7}=1$ being hold in the conditions of the differential path, we can compute bit 7 of $F_{3}\left(X_{36}, X_{35},\left(X_{34} \lll 10\right)\right)$, then $X_{37,21}$ can be calculated. Therefore, bits 17 and 21 of $X_{37}$ can be calculated correctly if the differential path hold.

The above procedure will definitely compute $X_{37, i}(i=17,21)$ correctly under the condition that $X_{36}$ is unknown, which is confirmed by the experiment. For the randomly chosen $X_{i}(i=32, \ldots, 36)$ and $m_{9}$ such that the above conditions are satisfied. On one hand, $X_{37}$ is calculated as

$$
X_{37}=\left(X_{33} \lll 10\right)+\left(\left(X_{32} \lll 10\right)+F_{3}\left(X_{36}, X_{35},\left(X_{34} \lll 10\right)\right)+m_{9}+k_{3}^{l}\right) \lll 14 .
$$

On the other hand, $X_{37}$ is computed as

$$
X_{37}=\left(X_{33} \lll 10\right)+\left(\left(X_{32} \lll 10\right)+\left(\left(\neg X_{34} \lll 10\right) \wedge \text { xef }\right)+\left(m_{9} \wedge \text { oxef }\right)+k_{3}^{l}\right) \lll 14
$$


The experiment shows that the two methods of calculation obtain the same value of $X_{37,17}$ $\left(X_{37,21}\right)$ with probability 1 , for randomly chosen $X_{36}$ which satisfies the conditions of the differential path. This means $X_{37,17}$ and $X_{37,21}$ can be computed correctly independent of $X_{36}$. The additional conditions of computing $X_{37, i}(i=2,7,17,21)$ and $X_{38, i}(i=17,21)$ are shown in Table 10. It is worth noting that these extra conditions are not contradictory to the conditions of the differential path.

2. $X_{37, i}(i=2,7)$ can be computed as follows. Let $X_{35, i}=0(i=17, \ldots, 25)$, then bits 17-25 of $F_{3}\left(X_{36}, X_{35},\left(X_{34} \lll 10\right)\right)$ can be computed. Let

$$
T_{2}=\left(X_{32} \lll 10\right)+\left(\left(\neg X_{34} \lll 10\right) \wedge 0 \times 3 f e 0000\right)+\left(m_{9} \wedge 0 \times 3 \mathrm{fe} 0000\right)+k_{3}^{l},
$$

and force $T_{2,17}=0$. Let $S_{1}=\left(X_{32} \lll 10\right)+k_{3}^{l}$, and add the conditions $S_{1, i}=0(i=16,17)$. Let $S_{2}=F_{3}\left(X_{36}, X_{35},\left(X_{34} \lll 10\right)\right)+m_{9}$, and force $S_{2,16}=0$. Then there will be no carry coming from bit 17 to bit 18 in the process of computing $T_{2}$. Therefore, the 8 lowest bits of $X_{37}$ especially $X_{37, i}(i=2,7)$ can be calculated correctly by

$$
\left(X_{33} \lll 10\right)+\left(\left(X_{32} \lll 10\right)+\left(\left(\neg X_{34} \lll 10\right) \wedge 0 \times 3 f e 0000\right)+m_{9}+k_{3}^{l}\right) \lll 14 .
$$

In [MPS $\left.{ }^{+} 13\right]$, in order to compute $X_{37, i}(i=2,7)$, the conditions that the 16 -th bit of $\left(X_{32} \lll\right.$ $10)+\left(F_{3}\left(X_{36}, X_{35},\left(X_{34} \lll 10\right)\right) \wedge 0 \times 3 \mathrm{ff0000}\right)+\left(m_{9} \wedge 0 \times 3 \mathrm{ff0000}\right)+k_{3}^{l}$ equals to zero and $X_{35, i}=0(i=17, \ldots, 25)$ are added. The experiment shows that the probability of $X_{37, i}(i=2,7)$ being computed correctly is less than 1 . It is due to the carry from bit 17 to bit 18 can not always be stopped when computing $\left(X_{32} \lll 10\right)+F_{3}\left(X_{36}, X_{35},\left(X_{34} \lll 10\right)\right)+m_{9}+k_{3}^{l}$.

Calculate $X_{38, i}(i=17,21)$

Since $F_{3}\left(X_{37}, X_{36},\left(X_{35} \lll 10\right)\right)=\left(X_{37} \vee \neg X_{36}\right) \oplus\left(X_{35} \lll 10\right)$ and $X_{36}$ is unknown, then the output of $F_{3}$ can be computed if $X_{37}=0 \times f f f f f f f f$. Taking into consideration the fact that

$$
X_{38}=\left(X_{34} \lll 10\right)+\left(\left(X_{33} \lll 10\right)+F_{3}\left(X_{37}, X_{36},\left(X_{35} \lll 10\right)\right)+m_{15}+k_{3}^{l}\right) \lll 9,
$$

we had better obtain bits $0-12$ of $F_{3}\left(X_{37}, X_{36},\left(X_{35} \lll 10\right)\right)$ in order to compute $X_{38, i}(i=17,21)$. Thus, we need to force $X_{37, i}=1(i=0, \ldots, 12)$. We have computed $X_{37, i}(i=0, \ldots, 7)$ above. Furthermore, $X_{37, i}(i=8, \ldots, 12)$ can be calculated in the same way as the above method by forcing $X_{35, i}=0(i=26, \ldots, 30)$. However, there is $X_{37,2}=0$ in the differential path. Thanks to $X_{36,2}=1$ in the conditions of differential path, so we can get bit 2 of $F_{3}\left(X_{37}, X_{36},\left(X_{35} \lll 10\right)\right)$. Thus, bits 0-12 of $F_{3}\left(X_{37}, X_{36},\left(X_{35} \lll 10\right)\right)$ can be obtained. Let

$$
T_{3}=\left(X_{33} \lll 10\right)+\left(\left(0 \times 1 \mathrm{ffb} \oplus\left(X_{35} \lll 10\right)\right) \wedge 0 \mathrm{x} 1 \mathrm{fff}\right)+\left(m_{15} \wedge 0 \mathrm{x} 1 \mathrm{fff}\right)+k_{3}^{l},
$$

and force $T_{3,7}=0$. Moreover, we add the extra condition $X_{34,6}=0$ by modifying $m_{10}$. Therefore, the carry will be stopped at bit 16 in the process of computing $X_{38}$. Thus, $X_{38, i}(i=17, \ldots, 21)$ can be calculated correctly by

$$
\left(X_{34} \lll 10\right)+\left(T_{3} \lll 9\right) .
$$

We verify the above computations by experiments, which show that $X_{37, i}(i=2,7,17,21)$ and $X_{38, i}(i=17,21)$ can be calculated correctly with probability 1 independent of $X_{36}$. Our C implementation verifying the computation of $X_{37, i}(i=2,7,17,21)$ and $X_{38, i}(i=17,21)$ can be found in [ver16a].

Moreover, we give an example in Table 5, where $X_{i}$ and $X_{i}^{\prime}(i=17,18,19,20,21)$ satisfy the conditions of the differential path in the left branch in Table 7. Then for randomly chosen $X_{36}$ and $X_{36}^{\prime}$ (which satisfy the conditions of the differential path), we can get that $X_{37, i}=0(i=2,21)$, $X_{37, i}=1(i=0,1,3, \ldots, 12,17), X_{38, i}=0(i=17,21), \Delta X_{37}=0 \times 200000$ and $\Delta X_{38}=0$ hold, which means $X_{37}, X_{37}^{\prime}, X_{38}$ and $X_{38}^{\prime}$ follow the differential path in Table 7. Therefore, the conditions 
Table 5: An Example of the 17-38 Steps Differential Path in the Left Branch

\begin{tabular}{|c|c|c|c|c|c|}
\hline $\begin{array}{c}X_{17} \\
\text { e17af3a8 } \\
X_{17}^{\prime} \\
\text { e17af3e8 }\end{array}$ & $\begin{array}{c}X_{18} \\
\operatorname{cc} 966 \mathrm{a} 05 \\
X_{18}^{\prime} \\
\operatorname{cc} 966 \mathrm{a} 05\end{array}$ & $\begin{array}{c}X_{19} \\
\text { b7573bfe } \\
X_{19}^{\prime} \\
\text { b7573bfe }\end{array}$ & $\begin{array}{c}X_{20} \\
9336419 \mathrm{e} \\
X_{20}^{\prime} \\
9336419 \mathrm{e}\end{array}$ & $\begin{array}{c}X_{21} \\
\text { f6920500 } \\
X_{21}^{\prime} \\
\text { f6930500 }\end{array}$ & $\begin{array}{l}X_{36}{ }^{c} \\
\mathrm{RV} \\
X_{36}^{\prime} \\
\mathrm{RV}^{\prime}\end{array}$ \\
\hline $\begin{array}{c}m_{0} \\
7 \mathrm{ed} 471 \mathrm{ba} \\
m_{9} \\
2 \mathrm{e} 63 \mathrm{c} 462\end{array}$ & $\begin{array}{c}m_{2} \\
\text { 7e } 3 \mathrm{~d} 71 \mathrm{~d} 1 \\
m_{10} \\
\text { b0 } 477764\end{array}$ & $\begin{array}{c}m_{3} \\
1 \mathrm{edb} 7709 \\
m_{11} \\
67740 \mathrm{efc}\end{array}$ & $\begin{array}{c}m_{5} \\
\text { a } 3 d 9 b 37 \mathrm{f} \\
m_{12} \\
\text { f } 9 \text { e } 7 \mathrm{~b} 23 \mathrm{c}\end{array}$ & $\begin{array}{c}m_{6} \\
70477536 \\
m_{14} \\
50321425\end{array}$ & $\begin{array}{c}m_{8} \\
\text { bdb } 9 \text { fa } 58 \\
m_{15} \\
61 \mathrm{~b} 8 \mathrm{f} 56 \mathrm{a}\end{array}$ \\
\hline \multicolumn{4}{|c|}{$\begin{array}{l}\quad\left(X_{37}, X_{37}^{\prime}\right) \\
X_{37, i}=0(i=2,21) \\
=1(i=0,1,3, \ldots, 12,17) \\
\Delta X_{37}=200000\end{array}$} & \multicolumn{2}{|c|}{$\begin{array}{l}\left(X_{38}, X_{38}^{\prime}\right) \\
=0(i=17,21)\end{array}$} \\
\hline
\end{tabular}

${ }^{c} X_{36}$ and $X_{36}^{\prime}$ can be random values (RV) which satisfy the conditions of the differential path.

The values in the table are in hexadecimal notation, and " $0 \mathrm{x}$ " is omitted because of limited space.

of $X_{37}, X_{37}^{\prime}, X_{38}$ and $X_{38}^{\prime}$ in Table 5 can be guaranteed to be hold even though $X_{36}$ is unknown. Our $\mathrm{C}$ implementation of verifying the instance in Table 5 can be found in [ver16c].

\section{Calculate $Y_{30,9}$}

We need to know $Y_{30,9}$ in the process of computing $Y_{32,20}$, so we calculate $Y_{30,9}$ in advance. Because $F_{4}\left(Y_{29}, Y_{28},\left(Y_{27} \lll 10\right)\right)=\left(Y_{29} \wedge\left(Y_{27} \lll 10\right)\right) \vee\left(Y_{28} \wedge \neg\left(Y_{27} \lll 10\right)\right)$ and $Y_{29}$ is unknown, if the condition $Y_{27}=0$ is added, then

$$
Y_{30}=\left(Y_{26} \lll 10\right)+\left(\left(Y_{25} \lll 10\right)+F_{4}\left(Y_{29}, Y_{28},\left(Y_{27} \lll 10\right)\right)+m_{9}+k_{2}^{r}\right) \lll 15
$$

can be calculated. It is obviously that bits $23,24,26$ of $F_{4}\left(Y_{29}, Y_{28},\left(Y_{27} \lll 10\right)\right)$ can be calculated by adding the extra conditions $Y_{27, i}=0(i=13,14,16)\left(Y_{27,15}=1\right.$ is a condition of the differential path). Let

$$
\begin{gathered}
T_{4}=\left(Y_{25} \lll 10\right)+\left(Y_{28} \wedge 0 \times 5800000\right)+m_{9}+k_{2}^{r}, \\
R_{1}=\left(Y_{25} \lll 10\right)+F_{4}\left(Y_{29}, Y_{28},\left(Y_{27} \lll 10\right)\right)+m_{9}+k_{2}^{r}, \quad Q_{1}=R_{1} \lll 15, \\
R_{2}=\left(Y_{25} \lll 10\right)+m_{9}+k_{2}^{r}, \quad R_{3}=F_{4}\left(Y_{29}, Y_{28},\left(Y_{27} \lll 10\right)\right),
\end{gathered}
$$

and force $T_{4, i}=0(i=23,24), R_{2, i}=0(i=23,24,25)$, and $R_{3,23}=0$ (which is equivalent to $Y_{28,23}=0$ and $\left.Y_{27,13}=0\right)$. Therefore, $R_{1, i}=T_{4, i}(i=24,26)$ can be calculated correctly because the carry is stopped at bit 23 in the process of computing $R_{1}$. Thus, $Q_{1, i}(i=7,9)$ can be computed correctly and $Q_{1,7}=0$. Furthermore, we add the extra condition $Y_{26, i}=0(i=29,30)$, which will stop the carry coming from the lower bits when computing $Y_{30,9}$ combined with the condition $Q_{1,7}=0$. Therefore, $Y_{30,9}$ can be calculated correctly by

$$
\left(Y_{26} \lll 10\right)+\left(\left(Y_{25} \lll 10\right)+\left(Y_{28} \wedge 0 \times 5800000\right)+m_{9}+k_{2}^{r}\right) \lll 15 .
$$

The process of computing $Y_{30, i}(i=15,21,27,30,31)$ and $Y_{32,20}$ can be seen in Appendix. The additional conditions of computing $Y_{30, i}(i=9,15,21,27,30,31)$ and $Y_{32,20}$ are shown in Table 11, which are not contradictory to the conditions of the differential path.

We verify the above computations by experiment, which show that even though $Y_{29}$ is unknown, $Y_{30, i}(i=9,15,21,27,30,31)$ can be calculated correctly with probability 1 , and $Y_{32,20}$ can be obtained correctly with a probability of more than 0.9 . Our $\mathrm{C}$ implementation verifying the computation of $Y_{30, i}(i=9,15,21,27,30,31)$ and $Y_{32,20}$ can be found in [ver16b].

Moreover, we give an example in Table 6, where $Y_{i}$ and $Y_{i}^{\prime}(i=17,18,19,20,21)$ satisfy the conditions of the differential path in the right branch in Table 7 . Then for randomly chosen 
Table 6: An Example of the 17-32 Steps Differential Path in the Right Branch

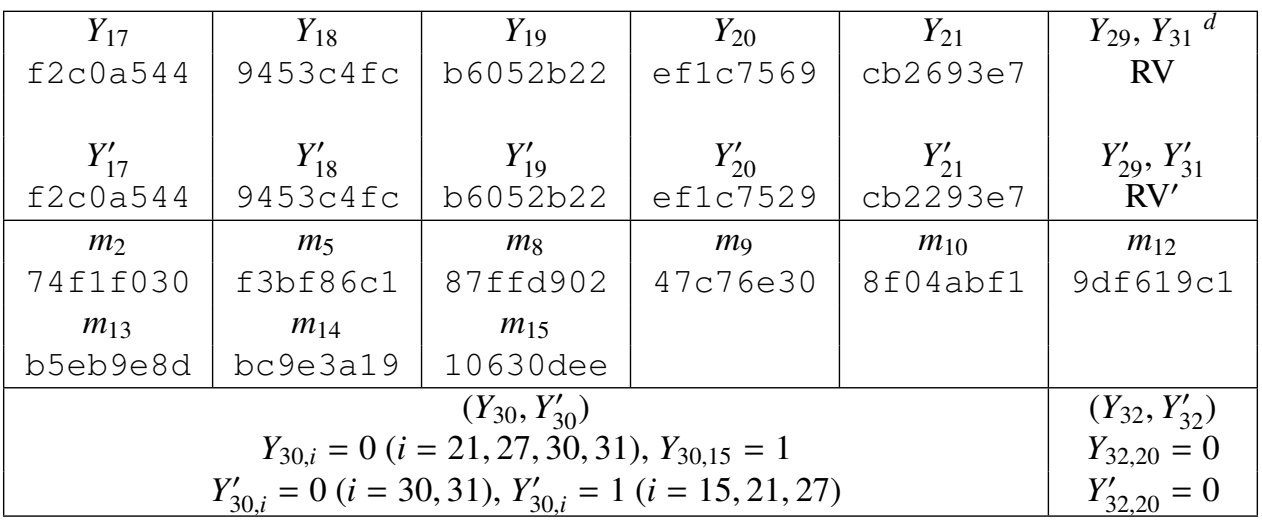

${ }^{d} Y_{29}, Y_{29}^{\prime}, Y_{31}$ and $Y_{31}^{\prime}$ can be random values (RV) which satisfy the conditions of the differential path. The values in the table are in hexadecimal notation, and "0x" is omitted because of limited space.

$Y_{29}, Y_{29}^{\prime}, Y_{31}$ and $Y_{31}^{\prime}$ (which satisfy the conditions of the differential path), we can get that $Y_{30, i}=0(i=21,27,30,31), Y_{30,15}=1, Y_{30, i}^{\prime}=0(i=30,31), Y_{30, i}^{\prime}=1(i=15,21,27)$ and $Y_{32,20}=Y_{32,20}^{\prime}=0$ hold with a probability of more than 0.9 . Therefore, the conditions of $Y_{30}, Y_{30}^{\prime}$, $Y_{32}$ and $Y_{32}^{\prime}$ in Table 6 can be guaranteed to be hold with a probability of more than 0.9 even though $Y_{29}$ and $Y_{31}$ are unknown. Our $\mathrm{C}$ implementation of verifying the instance in Table 6 can be found in [ver16d].

A starting point example is presented in Table 7, which follows the differential path in [MPS ${ }^{+} 13$ ]. Furthermore, for randomly chosen $X_{36}$ and $X_{36}^{\prime}$ which satisfy the conditions of the differential path, the starting point makes sure that $X_{37, i}=0(i=2,21), X_{37, i}=1(i=7,17), \Delta X_{37}=0 \times 200000$, $X_{38,17}=0$ and $\Delta X_{38}=0$ hold with probability 1 . Meanwhile, for randomly chosen $Y_{29}, Y_{29}^{\prime}$, $Y_{31}$ and $Y_{31}^{\prime}$ which satisfy the conditions of the differential path, the starting point ensures that $Y_{30, i}=0(i=21,27,30,31), Y_{30,15}=1$ and $\Delta Y_{30}=0 \times 8200000$ hold with probability 1 . Moreover, $Y_{32,20}=0$ holds with a probability of more than 0.9 even though $Y_{29}$ and $Y_{31}$ are unknown. Our C implementation of verifying the starting point in Table 7 can be found in [ver16e].

In this paper, one of the main works focuses on providing a method to compute the values of $X_{37, i}(i=2,7,17,21), X_{38, i}(i=17,21), Y_{30, i}(i=9,15,21,27,30,31)$ and $Y_{32,20}$ under the condition that $X_{36}, Y_{29}$ and $Y_{31}$ are unknown (these bits are listed in Table 4). Then the conditions on these bits are satisfied by message modification or exhaustive search. $Y_{30,9}$ is computed correctly in the starting point example shown in Table 7, and the purpose of computing $Y_{30,9}$ is to compute $Y_{32,20}$. It is noted that there is no condition on $Y_{30,9}$ in the differential path. There is a condition $X_{38,21}=0$ in the differential path. We had no high performance computer to make the condition $X_{38,21}=0$ hold. However, the value of $X_{38,21}$ is computed correctly (which is the most important part) in the starting point example shown in Table 7.

\section{Uncontrolled probability.}

1. After a starting point is discovered, the remaining free message words $m_{1}, m_{4}, m_{7}$ and $m_{13}$ are used to make sure that there is a perfect match on the values of the five initial words of both branches, i.e. $X_{i}=Y_{i}(i=12,13,14,15,16)$. Furthermore, the success probability of the match phase is $2^{-32}$.

2. In the left branch, after finding a starting point with the generalized message modification, there are 5 conditions $X_{36, i}=0(i=11,21), X_{36, i}=1(i=2,7,24)$ in Table 8 (except $X_{57}, \ldots$, $\left.X_{64}\right)$ that are not modified. Moreover, the uncontrolled probability of the left branch until step 56 is $2^{-5.4}$ by experiment. 


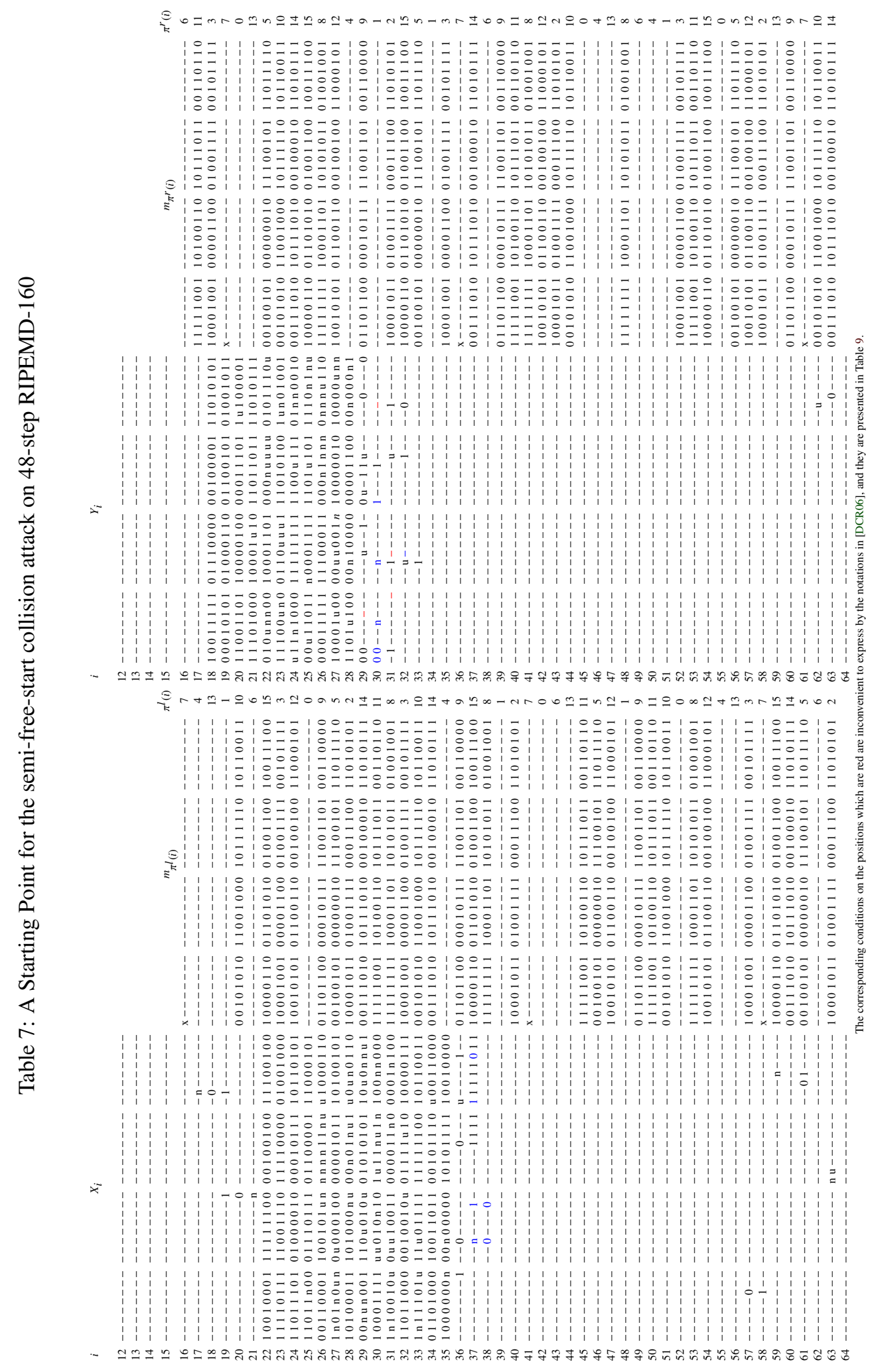


3. In the right branch, the conditions $Y_{30, i}(i=15,21,27,30,31)$ and $Y_{32,20}$ in Table 9 are satisfied in the starting points. Therefore, there are only 24 uncontrolled conditions between $Y_{29}$ and $Y_{33}$. Moreover, the uncontrolled probability of the right branch until step 59 is $2^{-29.6}$ by experiment.

4. The collision probability of the differential path in steps 57-64 of the left branch and in steps 60-64 of the right branch is $2^{-11.3}$ by experiment [MPS $\left.{ }^{+} 13\right]$.

Therefore, the uncontrolled probability is $2^{-32} \times 2^{-5.4} \times 2^{-29.6} \times 2^{-11.3} \times 0.9=2^{-78.5}$ in total.

\section{Complexity evaluation.}

1. The $2^{78.5}$ starting points do not need to be generated from the beginning. A new start point can be produced by randomizing $m_{6}$. Once all the possible choices of $m_{6}$ have been used, the freedom degrees of $m_{0}, m_{9}$ and $m_{5}$ can be used. According to Table 7, $m_{6}$ is used to fulfill one condition on $X_{17}$. In order to use the freedom degree of $m_{6}$, we can randomly choose $X_{17}$ satisfying the condition $X_{17,6}=0$ and deduce $m_{6}$. Therefore, the complexity of finding a new starting point from a known one is about $2 \div(48 \times 2) \approx 2^{-5.6}$ of the 48-step compression function. For randomizing $m_{0}, m_{9}$ and $m_{5}$, the corresponding number of times we have to regenerate them is not the bottleneck of the attack on 48-step RIPEMD-160. Therefore, the complexity of generating all the required starting points is $2^{78.5} \times 2^{-5.6}=2^{72.9}$.

2. The process of merging both branches is the same as the merging process proposed by Mendel et al. [MPS $\left.{ }^{+} 13\right]$. The values of $m_{13}$ and $m_{4}$ can be deduced from $X_{16}=Y_{16}$ and $X_{13}=Y_{13}$ respectively, and the complexity can be negligible. The bottleneck of the merging complexity is finding $m_{1}$ and $m_{7}$ from the conditions $X_{15}=Y_{15}$ and $X_{14}=Y_{14}$. The problem of finding the values of $m_{1}$ and $m_{7}$ is equivalent to solving the equation $X+C_{0}=\left(C_{1}+X \lll 8\right) \ggg 15$, where $C_{0}$ and $C_{1}$ are constants. The attackers pre-compute $m_{1}$ and $m_{7}$ and store them in a table for all the $2^{64}$ possible values of $C_{0}$ and $C_{1}$, with a time complexity of $2^{73}$ and memory complexity of $2^{64}$. Moreover, in the merging process, the table lookup is estimated using a RAM access and the implementation of one merging needs about 145 cycles, while the OPENSSL implementation of RIPEMD-160 compression function is about 1040 cycles. Therefore, one merging costs about $145 \div(1040 \times 48 \div 80) \approx 2^{-2.1}$ calls of the 48 -step compression function.

From the reasoning above, we can conclude that the complexity of the semi-free start collision attack on 48-step RIPEMD-160 is $2^{78.5-2.1}=2^{76.4}$.

\section{Conclusions}

In this paper, we present a method and give a partial answer to calculate the theoretical probability of a given differential path in RIPEMD-160, the step function of which is no longer a $T$-function. Furthermore, we propose a method to carry out the message modification such that a step differential path holds with probability 1 after message modification. Meanwhile, we propose a semi-free start collision attack on 48-step RIPEMD-160, and our result improves the previously best known semi-free start collision by 6 rounds. Our semi-free start collision attack uses the differential path constructed by Mendel et al. and improves the probabilistic part by a factor of about $2^{10}$. The future work includes completely answering the open problem of computing the theoretical probability of a given differential path in RIPEMD-160.

\section{Acknowledgments}

We would like to thank Florian Mendel and the anonymous reviewers who helped improve this paper. This work is supported by the National Natural Science Foundation of China (Nos. 61572125, 
61632012, 61373142), and Shanghai High-Tech Field Project (No. 16511101400).

\section{References}

[BP95] Antoon Bosselaers and Bart Preneel. Integrity Primitives for Secure Information Systems: Final Ripe Report of Race Integrity Primitives Evaluation. Number 1007. Springer Science \& Business Media, 1995.

[Dam89] Ivan Damgård. A design principle for hash functions. In Advances in Cryptology - CRYPTO '89, 9th Annual International Cryptology Conference, Santa Barbara, California, USA, August 20-24, 1989, Proceedings, pages 416-427, 1989.

[Dau05] Magnus Daum. Cryptanalysis of Hash functions of the MD4-family. PhD thesis, Ruhr-Universität Bochum, Universitätsbibliothek, 2005.

[DBP96] Hans Dobbertin, Antoon Bosselaers, and Bart Preneel. RIPEMD-160: A strengthened version of RIPEMD. In International Workshop on Fast Software Encryption, pages 71-82. Springer, 1996.

[DCR06] Christophe De Cannière and Christian Rechberger. Finding SHA-1 characteristics: General results and applications. In International Conference on the Theory and Application of Cryptology and Information Security, pages 1-20. Springer, 2006.

[DEM14] Christoph Dobraunig, Maria Eichlseder, and Florian Mendel. Analysis of SHA512/224 and SHA-512/256. In International Conference on the Theory and Application of Cryptology and Information Security, pages 612-630. Springer, 2014.

[Dob97] Hans Dobbertin. RIPEMD with two-round compress function is not collision-free. Journal of Cryptology, 10(1):51-69, 1997.

[FLN] Pierre-Alain Fouque, Gaetan Leurent, and Phong Nguyen. Automatic search of differential path in md4. ecrypt hash worshop-cryptology eprint archive, report, 2007/206 (2007).

[Int11] International Standardization of Organization (ISO). International Standard- ISO/IEC 29192-2, Information technology-Security techniques-Lightweight cryptography -Part 2: Block ciphers. 2011.

[Kli06] Vlastimil Klima. Tunnels in hash functions: Md5 collisions within a minute. IACR Cryptology ePrint Archive, 2006:105, 2006.

[LP13] Franck Landelle and Thomas Peyrin. Cryptanalysis of full ripemd-128. In Annual International Conference on the Theory and Applications of Cryptographic Techniques, pages 228-244. Springer, 2013.

[LP16] Franck Landelle and Thomas Peyrin. Cryptanalysis of full RIPEMD-128. Journal of Cryptology, 29(4):927-951, 2016.

[Mer89] Ralph C Merkle. One way hash functions and DES. In Conference on the Theory and Application of Cryptology, pages 428-446. Springer, 1989.

[MNS11] Florian Mendel, Tomislav Nad, and Martin Schläffer. Finding SHA-2 characteristics: searching through a minefield of contradictions. In International Conference on the Theory and Application of Cryptology and Information Security, pages 288-307. Springer, 2011. 
[MNS12] Florian Mendel, Tomislav Nad, and Martin Schläffer. Collision attacks on the reduced dual-stream hash function RIPEMD-128. In Fast Software Encryption, pages 226243. Springer, 2012.

[MNS13] Florian Mendel, Tomislav Nad, and Martin Schläffer. Improving local collisions: new attacks on reduced SHA-256. In Annual International Conference on the Theory and Applications of Cryptographic Techniques, pages 262-278. Springer, 2013.

[MNSS12] Florian Mendel, Tomislav Nad, Stefan Scherz, and Martin Schläffer. Differential attacks on reduced RIPEMD-160. In International Conference on Information Security, pages 23-38. Springer, 2012.

[MPRR06] Florian Mendel, Norbert Pramstaller, Christian Rechberger, and Vincent Rijmen. On the collision resistance of RIPEMD-160. In International Conference on Information Security, pages 101-116. Springer, 2006.

[MPS ${ }^{+}$13] Florian Mendel, Thomas Peyrin, Martin Schläffer, Lei Wang, and Shuang Wu. Improved cryptanalysis of reduced RIPEMD-160. In International Conference on the Theory and Application of Cryptology and Information Security, pages 484-503. Springer, 2013.

[OSS10] Chiaki Ohtahara, Yu Sasaki, and Takeshi Shimoyama. Preimage attacks on stepreduced RIPEMD-128 and RIPEMD-160. In International Conference on Information Security and Cryptology, pages 169-186. Springer, 2010.

[SHA] NIST SHA. SHA-3 cryptographic hash algorithm competition.

[SKP16] Marc Stevens, Pierre Karpman, and Thomas Peyrin. Freestart collision for full SHA-1. In Annual International Conference on the Theory and Applications of Cryptographic Techniques, pages 459-483. Springer, 2016.

[SW12] Yu Sasaki and Lei Wang. Distinguishers beyond three rounds of the RIPEMD-128/160 compression functions. In International Conference on Applied Cryptography and Network Security, pages 275-292. Springer, 2012.

[ver16a] Verification of the computation of $x_{37, i}(i=2,7,17,21)$ and $x_{38, i}(i=17,21) .2016$. https://github.com/Crypt-CNS/RIPEMD-160/tree/Reference1.

[ver16b] Verification of the computation of $y_{30, i}(i=9,15,21,27,30,31)$ and $y_{32,20} .2016$. https://github.com/Crypt-CNS/RIPEMD-160/tree/Reference2.

[ver16c] Verification of the instance in table 5. 2016. https://github.com/ Crypt-CNS/RIPEMD-160/tree/Reference3.

[ver16d] Verification of the instance in table 6. 2016. https://githul.com/ Crypt-CNS/RIPEMD-160/tree/Reference4.

[ver16e] Verification of the instance in table 7. 2016. https://github.com/ Crypt-CNS/RIPEMD-160/tree/Reference5.

[Wan14] Gaoli Wang. Practical collision attack on 40-step RIPEMD-128. In Cryptographers' Track at the RSA Conference, pages 444-460. Springer, 2014.

$\left[\mathrm{WLF}^{+} 05\right]$ Xiaoyun Wang, Xuejia Lai, Dengguo Feng, Hui Chen, and Xiuyuan Yu. Cryptanalysis of the hash functions MD4 and RIPEMD. In Annual International Conference on the Theory and Applications of Cryptographic Techniques, pages 1-18. Springer, 2005. 
[WS14] Gaoli Wang and Yanzhao Shen. (pseudo-) preimage attacks on step-reduced HAS160 and RIPEMD-160. In International Conference on Information Security, pages 90-103. Springer, 2014.

[WW08] Gaoli Wang and Meiqin Wang. Cryptanalysis of reduced RIPEMD-128. Journal of Software, 19(9):2442-2448, 2008.

[WY05] Xiaoyun Wang and Hongbo Yu. How to break MD5 and other hash functions. In Annual International Conference on the Theory and Applications of Cryptographic Techniques, pages 19-35. Springer, 2005.

[WY15] Gaoli Wang and Hongbo Yu. Improved cryptanalysis on RIPEMD-128. IET Information Security, 9(6):354-364, 2015.

[WYY05a] Xiaoyun Wang, Yiqun Lisa Yin, and Hongbo Yu. Finding collisions in the full SHA-1. In Annual International Cryptology Conference, pages 17-36. Springer, 2005.

[WYY05b] Xiaoyun Wang, Hongbo Yu, and Yiqun Lisa Yin. Efficient collision search attacks on SHA-0. In Annual International Cryptology Conference, pages 1-16. Springer, 2005.

[YWYP06] Hongbo Yu, Xiaoyun Wang, Aaram Yun, and Sangwoo Park. Cryptanalysis of the full HAVAL with 4 and 5 passes. In International Workshop on Fast Software Encryption, pages 89-110. Springer, 2006.

[YWZW05] Hongbo Yu, Gaoli Wang, Guoyan Zhang, and Xiaoyun Wang. The second-preimage attack on MD4. In International Conference on Cryptology and Network Security, pages 1-12. Springer, 2005. 


\section{A Some propositions of computing the differential proba- bility}

Proposition 2 Let $y=x_{1}+\left(x_{2}+x_{3}\right) \lll s, y^{\prime}=x_{1}+\left(x_{2}+x_{3}^{\prime}\right) \lll s$, here $x_{1}, x_{2}, x_{3}$ and $x_{3}^{\prime}$ are 32-bit words. If $\Delta x_{3}=[i, j]$ (i.e., $x_{3, i}=0, x_{3, j}=0, x_{3, i}^{\prime}=1, x_{3, j}^{\prime}=1, x_{3, k}=x_{3, k}^{\prime}(0 \leq k \leq 31, k \neq i, j)$ ) and $i<j \leq 31-s$, then the probability that $\Delta^{+} y$ equals to $2^{i+s}+2^{j+s}$ can be computed as follows:

$$
\operatorname{Pr}\left[\Delta^{+} y=2^{i+s}+2^{j+s}\right]=1-\frac{1}{2^{32-s-i}}-\frac{1}{2^{32-s-j}} .
$$

Proof. Let $r_{1}=x_{2}+x_{3}, r_{2}=r_{1} \lll s, r_{1}^{\prime}=x_{2}+x_{3}^{\prime}, r_{2}^{\prime}=r_{1}^{\prime} \lll s$, if the $i$-th bit difference of $\Delta x_{3}$ results in

$$
\begin{aligned}
\Delta r_{1} & =[i], \\
\text { or } \Delta r_{1} & =[-i, i+1], \\
\text { or } \Delta r_{1} & =[-i,-(i+1), i+2], \\
& \cdots \\
\text { or } \Delta r_{1} & =[-i,-(i+1), \ldots,-(j-2), j-1],
\end{aligned}
$$

and the $j$-th bit difference of $\Delta x_{3}$ results in

$$
\begin{aligned}
\Delta r_{1} & =[j], \\
\text { or } \Delta r_{1} & =[-j, j+1], \\
\text { or } \Delta r_{1} & =[-j,-(j+1), j+2], \\
& \cdots \\
\text { or } \Delta r_{1} & =[-j,-(j+1), \ldots,-(30-s), 31-s],
\end{aligned}
$$

then $\Delta^{+} y=2^{i+s}+2^{j+s}$ hold obviously.

Meanwhile, besides the above circumstances, it is not difficult to observe that $\Delta^{+} r_{2}=2^{i+s}+2^{j+s}$ (i.e., $\Delta^{+} y=2^{i+s}+2^{j+s}$ ) is equivalent to

$$
\begin{aligned}
\Delta r_{1} & =[-i,-(i+1), \ldots,-(j-1), j+1], \\
\text { or } \Delta r_{1} & =[-i,-(i+1), \ldots,-(j-1),-(j+1), j+2], \\
& \ldots \\
\text { or } \Delta r_{1} & =[-i,-(i+1), \ldots,-(j-1),-(j+1),-(j+2), \ldots,-(30-s), 31-s],
\end{aligned}
$$

Therefore,

$$
\begin{aligned}
& \operatorname{Pr}\left[\Delta^{+} y=2^{i+s}+2^{j+s}\right] \\
= & \left(\frac{1}{2}+\frac{1}{4}+\ldots+\frac{1}{2^{j-i}}\right) \times\left(\frac{1}{2}+\frac{1}{4}+\ldots+\frac{1}{2^{32-s-j}}\right)+\frac{1}{2^{j-i+1}}+\frac{1}{2^{j-i+2}}+\ldots+\frac{1}{2^{31-s-i}} \\
= & 1-\frac{1}{2^{32-s-i}}-\frac{1}{2^{32-s-j}} .
\end{aligned}
$$

From Proposition 2, it is easy to get the following corollary.

Corollary 2 Let $y=x_{1}+\left(x_{2}+x_{3}\right) \lll s, y^{\prime}=x_{1}+\left(x_{2}+x_{3}^{\prime}\right) \lll s$, here $x_{1}, x_{2}, x_{3}$ and $x_{3}^{\prime}$ are 32-bit words. If $\Delta x_{3}=[-i,-j]$ (i.e., $x_{3, i}=1, x_{3, j}=1, x_{3, i}^{\prime}=0, x_{3, j}^{\prime}=0, x_{3, k}=x_{3, k}^{\prime}(0 \leq k \leq 31, k \neq i, j)$ ) and $i<j \leq 31-s$, then the probability that $\Delta^{+} y$ equals to $-2^{i+s}-2^{j+s}$ can be computed as follows:

$$
\operatorname{Pr}\left[\Delta^{+} y=-2^{i+s}-2^{j+s}\right]=1-\frac{1}{2^{32-s-i}}-\frac{1}{2^{32-s-j}} .
$$


Proposition 3 Let $y=x_{1}+\left(x_{2}+x_{3}\right) \lll s, y^{\prime}=x_{1}+\left(x_{2}+x_{3}^{\prime}\right) \lll s$, here $x_{1}, x_{2}, x_{3}$ and $x_{3}^{\prime}$ are 32-bit words. If $\Delta x_{3}=[i, j]$ (i.e., $x_{3, i}=0, x_{3, j}=0, x_{3, i}^{\prime}=1, x_{3, j}^{\prime}=1, x_{3, k}=x_{3, k}^{\prime}(0 \leq k \leq 31, k \neq i, j)$ ) and $32 \leq i+s<j+s$, then the probability that $\Delta^{+} y$ equals to $2^{i+s-32}+2^{j+s-32}$ can be computed as follows:

$$
\operatorname{Pr}\left[\Delta^{+} y=2^{i+s-32}+2^{j+s-32}\right]=1-\frac{1}{2^{32-i}}-\frac{1}{2^{32-j}} .
$$

Proof. Let $r_{1}=x_{2}+x_{3}, r_{1}^{\prime}=x_{2}+x_{3}^{\prime}$, from $\Delta^{+} y=2^{i+s-32}+2^{j+s-32}$, we can get that the $i$-th bit difference of $\Delta x_{3}$ results in

$$
\begin{aligned}
\Delta r_{1} & =[i], \\
\text { or } \Delta r_{1} & =[-i, i+1], \\
\text { or } \Delta r_{1} & =[-i,-(i+1), i+2], \\
& \cdots \\
\text { or } \Delta r_{1} & =[-i,-(i+1), \ldots,-30,31],
\end{aligned}
$$

and the $j$-th bit difference of $\Delta x_{3}$ results in

$$
\begin{aligned}
\Delta r_{1} & =[j], \\
\text { or } \Delta r_{1} & =[-j, j+1], \\
\text { or } \Delta r_{1} & =[-j,-(j+1), j+2], \\
& \cdots \\
\text { or } \Delta r_{1} & =[-j,-(j+1), \ldots,-30,31] .
\end{aligned}
$$

It is obvious that the above circumstances except for $\Delta r_{1}=[-i,-(i+1), \ldots,-30,31]$ (from the $i$-th bit difference of $\left.\Delta x_{3}\right)$ and $\Delta r_{1}=[-j,-(j+1), \ldots,-30,31]$ (from the $j$-th bit difference of $\Delta x_{3}$ ) will lead to $\Delta^{+} y=2^{i+s-32}+2^{j+s-32}$. Therefore,

$$
\begin{aligned}
& \operatorname{Pr}\left[\Delta^{+} y=2^{i+s-32}+2^{j+s-32}\right] \\
= & \left(\frac{1}{2}+\frac{1}{2^{2}}+\frac{1}{2^{3}}+\ldots+\frac{1}{2^{32-i}}\right) \times\left(\frac{1}{2}+\frac{1}{2^{2}}+\frac{1}{2^{3}}+\ldots+\frac{1}{2^{32-j}}\right)-\frac{1}{2^{32-i}} \times \frac{1}{2^{32-j}} \\
= & \left(1-\frac{1}{2^{32-i}}\right) \times\left(1-\frac{1}{2^{32-j}}\right)-\frac{1}{2^{32-i}} \times \frac{1}{2^{32-j}} \\
= & 1-\frac{1}{2^{32-i}}-\frac{1}{2^{32-j}} .
\end{aligned}
$$

Proposition 4 For the $i$-th step function $X_{i}=\left(X_{i-4} \lll 10\right)+\left(\left(X_{i-5} \lll 10\right)+f\left(X_{i-1}, X_{i-2},\left(X_{i-3} \lll\right.\right.\right.$ $10))+m+k) \lll s$, where $m, k$ and $s$ depend on the step $i$. If the input difference is $\left(\Delta X_{i-5}\right.$, $\left.\Delta X_{i-4}, \Delta X_{i-3}, \Delta X_{i-2}, \Delta X_{i-1}\right)=(0,0,0,0,0)$, and the message difference $\Delta m=[j]$, then there are the following properties:

1. When $j+s \leq 31$, the probability of $\Delta X_{i}=[-(j+s),-(j+s+1), \ldots,-(j+s+t-1), j+s+t]$ is $\frac{1}{2^{t+1}} \times\left(1-\frac{1}{2^{32-s-j}}\right)$, where $j+s+t \leq 31$.

2. When $j+s>31$, the probability of $\Delta X_{i}=[-(j+s-32),-(j+s+1-32), \ldots,-(j+s+t-$ $1-32), j+s+t-32]$ is $\frac{1}{2^{t+1}} \times\left(1-\frac{1}{2^{32-j}}\right)$, where $j+s+t-32 \leq 31$.

Especially, when $t=0$, the probability of $\Delta X_{i}=[j+s(\bmod 32)]$ is $\frac{1}{2} \times\left(1-\frac{1}{2^{32-s-j}}\right)(j+s \leq 31)$ or $\frac{1}{2} \times\left(1-\frac{1}{2^{32-j}}\right)(j+s>31)$. 
Proof. (1) When $j+s \leq 31$,

$$
\Delta X_{i}=[-(j+s),-(j+s+1), \ldots,-(j+s+t-1), j+s+t] \Longleftrightarrow\left\{\begin{array}{l}
\Delta^{+} X_{i}=2^{j+s} \\
X_{i, j+s}=1 \\
X_{i, j+s+1}=1 \\
\cdots \\
X_{i, j+s+t-1}=1 \\
X_{i, j+s+t}=0
\end{array}\right.
$$

Combined with Proposition 1, we can get the probability of $\Delta X_{i}=[-(j+s),-(j+s+1), \ldots,-(j+$ $s+t-1), j+s+t]$ is $\frac{1}{2^{t+1}} \times\left(1-\frac{1}{2^{32-s-j}}\right)$.

(2) When $j+s>31$,

$$
\begin{array}{r}
\Delta X_{i}=[-(j+s-32),-(j+s+1-32), \ldots,-(j+s+t-1-32), j+s+t-32] \\
\Longleftrightarrow\left\{\begin{array}{c}
\Delta^{+} X_{i}=2^{j+s-32} \\
X_{i, j+s-32}=1 \\
X_{i, j+s+1-32}=1 \\
\cdots \\
X_{i, j+s+t-1-32}=1 \\
X_{i, j+s+t-32}=0
\end{array}\right.
\end{array}
$$

Combined with Proposition 1, we can get the probability of $\Delta X_{i}=[-(j+s-32),-(j+s+1-$ $32), \ldots,-(j+s+t-1-32), j+s+t-32]$ is $\frac{1}{2^{t+1}} \times\left(1-\frac{1}{2^{32-j}}\right)$.

\section{B The second example to illustrate the process of message modification}

The first example of the message modification is shown in Section 2.2. Following is the second example to illustrate the message modification.

Example 2. For the $i$-th step function $X_{i}=\left(X_{i-4} \lll 10\right)+\left(\left(X_{i-5} \lll 10\right)+f\left(X_{i-1}, X_{i-2},\left(X_{i-3} \lll\right.\right.\right.$ $10))+m+k) \lll s$, where $m, k$ and $s$ depend on the step $i$. Denote $r_{1}=\left(X_{i-5} \lll 10\right)+$ $f\left(X_{i-1}, X_{i-2},\left(X_{i-3} \lll 10\right)\right)+m+k, r_{1}^{\prime}=\left(X_{i-5} \lll 10\right)+f\left(X_{i-1}, X_{i-2},\left(X_{i-3} \lll 10\right)\right)+m^{\prime}+k$, $r_{2}=r_{1} \lll s$ and $r_{2}^{\prime}=r_{1}^{\prime} \lll s$. Let $\Delta m=$ [26] and $s=3$, in order to ensure $\Delta X_{i}=$ [29], the message modification can be processed as follows.

Step 1. If $\Delta^{+} X_{i} \neq 2^{29}$, from the proof of Proposition 1, we know that $\Delta r_{1}=[-26,-27,-28,29]$, $[-26,-27,-28,-29,30],[-26,-27,-28,-29,-30,31]$ or $[-26,-27,-28,-29,-30,-31]$. In order to ensure $\Delta^{+} X_{i}=2^{29}$, the message word $m$ can be modified as $m \longleftarrow m \pm 2^{28}, m \longleftarrow m \pm 2^{27}$ or $m \longleftarrow m \pm 2^{26}$. The reasons are explained as follows.

(1) If $r_{1,31}=1$ and $r_{1,31}^{\prime}=0$, in order to make $\Delta^{+} X_{i}=2^{29}, m$ can be modified in the following ways:

(1) The message word $m$ can be modified as $m \longleftarrow m-2^{28}$, then the most six significant bits of $r_{1}$ and $r_{1}^{\prime}$ are $r_{1, t}=1(t=26,27,29,30,31), r_{1,28}=0, r_{1, t}^{\prime}=1(t=28,29,30,31)$, and $r_{1, t}^{\prime}=0(t=26,27)$, which means $\Delta r_{1}=[-26,-27,28]$, thus $\Delta r_{2}=[-29,-30,31]$. Therefore, $\Delta^{+} X_{i}=-2^{29}-2^{30}+2^{31}=2^{29}$.

(2) Similarly, $m$ can be modified as $m \longleftarrow m+2^{28}$, then $r_{1, t}=1(t=26,27), r_{1, t}=0(t=$ $28,29,30,31), r_{1, t}^{\prime}=0(t=26,27,29,30,31)$, and $r_{1,28}^{\prime}=1$, which means $\Delta r_{1}=[-26,-27,28]$, thus $\Delta^{+} X_{i}=2^{29}$. 
(3) The message word $m$ can be modified as $m \longleftarrow m-2^{27}$, then the most six significant bits of $r_{1}$ and $r_{1}^{\prime}$ are $r_{1, t}=1(t=26,28,29,30,31), r_{1,27}=0, r_{1, t}^{\prime}=1(t=27,28,29,30,31)$, and $r_{1,26}^{\prime}=0$, which means $\Delta r_{1}=[-26,27]$, thus $\Delta r_{2}=[-29,30]$. Therefore, $\Delta^{+} X_{i}=-2^{29}+2^{30}=2^{29}$.

(4) Similarly, $m$ can be modified as $m \longleftarrow m+2^{27}$, then $r_{1, t}=0(t=27,28,29,30,31), r_{1,26}=1$, $r_{1, t}^{\prime}=0(t=26,28,29,30,31)$, and $r_{1,27}^{\prime}=1$, which means $\Delta r_{1}=[-26,27]$, thus $\Delta^{+} X_{i}=2^{29}$.

(5) The message word $m$ can be modified as $m \longleftarrow m-2^{26}$, then the most six significant bits of $r_{1}$ and $r_{1}^{\prime}$ are $r_{1, t}=1(t=27,28,29,30,31), r_{1,26}=0$ and $r_{1, t}^{\prime}=1(t=26,27,28,29,30,31)$, which means $\Delta r_{1}=[26]$, thus $\Delta r_{2}=[29]$. Therefore, $\Delta^{+} X_{i}=2^{29}$.

(6) Similarly, $m$ can be modified as $m \longleftarrow m+2^{26}$, then $r_{1, t}=0(t=26,27,28,29,30,31)$, $r_{1,26}^{\prime}=1$ and $r_{1, t}^{\prime}=0(t=27,28,29,30,31)$, which means $\Delta r_{1}=[26]$, thus $\Delta^{+} X_{i}=2^{29}$.

(2) If $\Delta r_{1}$ equals to $[-26,-27,-28,29]$, [ $\left.-26,-27,-28,-29,30\right]$ or $[-26,-27,-28,-29,-30,31]$, similar to Step (1), the message word $m$ can be modified according to one of the following three ways: $m \longleftarrow m \pm 2^{28}, m \longleftarrow m \pm 2^{27}$ or $m \longleftarrow m \pm 2^{26}$. After the message modification, we will get $\Delta r_{1}=[-26,-27,28], \Delta r_{1}=[-26,27]$ or $\Delta r_{1}=[26]$, respectively. Thus $\Delta^{+} X_{i}=2^{29}$ can be obtained.

Step 2. After Step 1 , we can get $\Delta^{+} X_{i}=2^{29}$, which means $\Delta r_{1}$ equals to [26], [-26, 27] or $[-26,-27,28]$. If $X_{i, 29} \neq 0$, the message word $m$ can be modified as $m \longleftarrow m+2^{26}$ or $m \longleftarrow m-2^{26}$ according to different circumstances. We give two examples to illustrate the modification.

(1) If $r_{1,31 \sim 26}^{\prime}=000001$ and $r_{1,31 \sim 26}=000000$, then the modification of $m$ as $m \longleftarrow m-2^{26}$ will violate the condition $\Delta^{+} X_{i}=2^{29}$. Therefore, $m$ can only be modified as $m \longleftarrow m+2^{26}$. This modification will ensure $\Delta^{+} X_{i}=2^{29}$ and $X_{i, 29}$ is flipped.

(2) If $r_{1,28 \sim 26}^{\prime}=101$ and $r_{1,28 \sim 26}=100$, then $m$ can be modified as $m \longleftarrow m+2^{26}$ or $m \longleftarrow m-2^{26}$. Both modification will ensure $\Delta^{+} X_{i}=2^{29}$ and $X_{i, 29}$ is flipped.

\section{The Process of Computing $Y_{30, i}(i=15,21,27,30,31)$ and $Y_{32,20}$}

\section{C.1 The Process of Computing $Y_{30,15}$}

$Y_{29}$ is unknown, from $F_{4}\left(Y_{29}, Y_{28},\left(Y_{27} \lll 10\right)\right)=\left(Y_{29} \wedge\left(Y_{27} \lll 10\right)\right) \vee\left(Y_{28} \wedge \neg\left(Y_{27} \lll 10\right)\right)$ we can get that bit 0 of $F_{4}\left(Y_{29}, Y_{28},\left(Y_{27} \lll 10\right)\right)$ can be calculated by adding the extra conditions $Y_{27,22}=0$. Meanwhile, $Y_{29, i}=0(i=30,31)$ hold in the conditions of the differential path, which will make bits 30,31 of $F_{4}\left(Y_{29}, Y_{28},\left(Y_{27} \lll 10\right)\right)$ can be calculated. Let

$$
\begin{gathered}
T_{5}=\left(Y_{25} \lll 10\right)+\left(Y_{28} \wedge\left(\neg Y_{27} \lll 10\right) \wedge 0 \times 00000001\right)+m_{9}+k_{2}^{r}, \\
R_{4}=\left(Y_{25} \lll 10\right)+F_{4}\left(Y_{29}, Y_{28},\left(Y_{27} \lll 10\right)\right)+m_{9}+k_{2}^{r}, \quad Q_{4}=R_{4} \lll 15,
\end{gathered}
$$

and force $T_{5, i}=0(i=30,31)$. Then bits 0,31 of $R_{4}$ can be calculated correctly, which means bits 14,15 of $Q_{4}$ can be computed correctly. Furthermore, we add the extra condition $Y_{26,4}=0$ (which has been hold in the differential path), which will make bit 15 of

$$
Y_{30}=\left(Y_{26} \lll 10\right)+\left(\left(Y_{25} \lll 10\right)+F_{4}\left(Y_{29}, Y_{28},\left(Y_{27} \lll 10\right)\right)+m_{9}+k_{2}^{r}\right) \lll 15
$$

can be calculated correctly by

$$
\left(Y_{26} \lll 10\right)+\left(\left(Y_{25} \lll 10\right)+\left(Y_{28} \wedge\left(\neg Y_{27} \lll 10\right) \wedge 0 \times c 0000001\right)+m_{9}+k_{2}^{r}\right) \lll 15
$$


since the carry is stopped in bit 14 when computing $Y_{30}$.

\section{C.2 The Process of Computing $Y_{30,21}$}

$Y_{29}$ is unknown, then bit 6 of $F_{4}\left(Y_{29}, Y_{28},\left(Y_{27} \lll 10\right)\right)$ can be calculated by adding the extra condition $Y_{27,28}=0$. There is $Y_{27,27}=1$ in the differential path, so bit 5 of $F_{4}\left(Y_{29}, Y_{28},\left(Y_{27} \lll 10\right)\right)$ can not be calculated. From $Y_{28,4}=0$ and $Y_{29,4}=0$ in the differential path, we can get that bit 4 of $F_{4}\left(Y_{29}, Y_{28},\left(Y_{27} \lll 10\right)\right)$ equals to zero. Similarly, by adding the conditions $Y_{27,25}=0$ and $Y_{28,3}=0$, we can get that bit 3 of $F_{4}\left(Y_{29}, Y_{28},\left(Y_{27} \lll 10\right)\right)$ equals to zero. Let

$$
R_{5}=\left(Y_{25} \lll 10\right)+m_{9}+k_{2}^{r},
$$

and force $R_{5, i}=0(i=3,4,5)$, then there is no carry from bit 3 to 4 when computing $R_{6}$, and $R_{6,4}=0, Q_{6,19}=0$, where

$$
R_{6}=\left(Y_{25} \lll 10\right)+F_{4}\left(Y_{29}, Y_{28},\left(Y_{27} \lll 10\right)\right)+m_{9}+k_{2}^{r}, \quad Q_{6}=R_{6} \lll 15 .
$$

Combined with the conditions $Y_{26, i}=0(i=9,10)$ (which have been hold in the differential path), $Y_{30,21}$ can be calculated correctly by

$$
\left(Y_{26} \lll 10\right)+\left(\left(Y_{25} \lll 10\right)+\left(Y_{28} \wedge\left(\neg Y_{27} \lll 10\right) \wedge 0 \times 78\right)+m_{9}+k_{2}^{r}\right) \lll 15
$$

because the carry can be stopped at bit 20 when computing $Y_{30}$.

\section{C.3 The Process of Computing $Y_{30, i}(i=27,30,31)$}

$Y_{29}$ has no influence on the bits $10,11,13, \ldots, 16$ of $F_{4}\left(Y_{29}, Y_{28},\left(Y_{27} \lll 10\right)\right)$ by adding the conditions $Y_{27, i}=0(i=0,1,3, \ldots, 6)$ (in fact, $Y_{27, i}=0(i=0,1)$ already hold in the conditions of the differential path). Thanks to the condition $Y_{29,12}=1$ in the differential path, we can compute bit 12 of $F_{4}\left(Y_{29}, Y_{28},\left(Y_{27} \lll 10\right)\right)$. Let

$$
T_{7}=\left(Y_{25} \lll 10\right)+\left(\left(Y_{28} \wedge 0 \times 1 \mathrm{fc} 00\right) \vee 0 \times 1000\right)+m_{9}+k_{2}^{r},
$$

and force $T_{7,10}=0$ and $T_{7,11}=1$. Let

$$
R_{7}=\left(Y_{25} \lll 10\right)+F_{4}\left(Y_{29}, Y_{28},\left(Y_{27} \lll 10\right)\right)+m_{9}+k_{2}^{r}, \quad Q_{7}=R_{7} \lll 15,
$$

thus, $R_{7, i}(i=11, \ldots, 16)$ can be obtained correctly since the carry is stopped in bit 10 when computing $R_{7}$. Obviously, $Q_{7, i}(i=26, \ldots, 31)$ can be obtained correctly and $Q_{7,26}=1$ hold. Combined with the condition $Y_{26,16}=1$ (which has been satisfied in the conditions of the differential path), we can deduce that $Y_{30, i}(i=27, \ldots, 31)$ can be obtained correctly by

$$
\left(Y_{26} \lll 10\right)+\left(\left(Y_{25} \lll 10\right)+\left(\left(Y_{28} \wedge 0 \times 1 \mathrm{fC} 00\right) \vee 0 \times 1000\right)+m_{9}+k_{2}^{r}\right) \lll 15
$$

since there is always a carry in bit 26 when computing $Y_{30}$.

\section{C.4 The Process of Computing $Y_{32,20}$}

From the step function, we know that

$$
Y_{32}=\left(Y_{28} \lll 10\right)+\left(\left(Y_{27} \lll 10\right)+F_{4}\left(Y_{31}, Y_{30},\left(Y_{29} \lll 10\right)\right)+m_{2}+k_{2}^{r}\right) \lll 11 .
$$

There is $Y_{29,31}=0$ in the conditions of the differential path, which makes $Y_{31,9}$ has no influence on the output of the 9th bit of $F_{4}\left(Y_{31}, Y_{30},\left(Y_{29} \lll 10\right)\right)$. Meanwhile, we can compute $Y_{30,9}$ in the 
Table 8: The Conditions of Steps 36-38, 57-64 in Left Branch

\begin{tabular}{|c|l|}
\hline $\begin{array}{c}\text { Chaining } \\
\text { Variable }\end{array}$ & Conditions on the Chaining Variable \\
\hline$X_{36}$ & $X_{36, i}=0(i=11,21), X_{36, i}=1(i=2,7,24)$ \\
\hline$X_{37}$ & $X_{37, i}=0(i=2,21), X_{37, i}=1(i=7,17)$ \\
\hline$X_{38}$ & $X_{38, i}=0(i=17,21)$ \\
\hline$X_{57}$ to $X_{59}$ & $X_{57,26}=0, X_{58,26}=1, X_{59,4}=0$ \\
\hline$X_{61}$ to $X_{63}$ & $X_{61,4}=1, X_{61,5}=0, X_{61,14}=X_{60,14}, X_{63,14}=1, X_{63,15}=0$ \\
\hline
\end{tabular}

Table 9: The Conditions of Steps 29-33, 60-64 in Right Branch

\begin{tabular}{|c|l|}
\hline $\begin{array}{c}\text { Chaining } \\
\text { Variable }\end{array}$ & Conditions on the Chaining Variable \\
\hline$Y_{29}$ & $\begin{array}{l}Y_{29, i}=0(i=0,4,15,30,31), Y_{29,26}=Y_{28,26}, \\
Y_{29, i}=1(i=10,11,12,14,17,20)\end{array}$ \\
\hline$Y_{30}$ & $Y_{30, i}=0(i=21,27,30,31), Y_{30, i}=1(i=11,15), Y_{30,5}=Y_{29,5}$ \\
\hline$Y_{31}$ & $Y_{31, i}=1(i=5,10,21,30), Y_{31,20} \neq Y_{30,20}, Y_{31,24}=Y_{30,24}$ \\
\hline$Y_{32}$ & $Y_{32,5}=0, Y_{32, i}=1(i=10,21)$ \\
\hline$Y_{33}$ & $Y_{33,21}=1, Y_{33,20}=1$ or $Y_{32,20}=0$ \\
\hline$Y_{60}$ to $Y_{64}$ & $Y_{61,4}=Y_{60,26}, Y_{62,4}=1, Y_{63,4}=0$ \\
\hline
\end{tabular}

previous computations. Therefore, the output of bit 9 of $F_{4}\left(Y_{31}, Y_{30},\left(Y_{29} \lll 10\right)\right)$ can be obtained. Let

$$
\begin{gathered}
T_{8}=\left(Y_{27} \lll 10\right)+\left(Y_{30} \wedge 0 \times 200\right)+m_{2}+k_{2}^{r}, \\
R_{8}=\left(Y_{27} \lll 10\right)+F_{4}\left(Y_{31}, Y_{30},\left(Y_{29} \lll 10\right)\right)+m_{2}+k_{2}^{r}, \quad Q_{8}=R_{8} \lll 11, \\
R_{9}=\left(Y_{27} \lll 10\right)+m_{2}+k_{2}^{r},
\end{gathered}
$$

and force $R_{9, i}=0(i=6,7,8)$.

Moreover, we add the conditions that $Y_{28, i}=0(i=6,7,8,9)$, then $Y_{32,20}$ can be obtained correctly with a probability of more than 0.9 by

$$
\left(Y_{28} \lll 10\right)+\left(\left(Y_{27} \lll 10\right)+\left(Y_{30} \wedge 0 \times 200\right)+m_{2}+k_{2}^{r}\right) \lll 11 .
$$

\section{The conditions on some chaining variables}

Table 10: The Extra Conditions of the Generalized Message Modification in the Left Branch

\begin{tabular}{|c|l|}
\hline $\begin{array}{c}\text { Chaining } \\
\text { Variable }\end{array}$ & Extra Conditions on the Chaining Variable \\
\hline$X_{33}$ & $X_{33, i}=0(i=6,8,9)$ \\
\hline$X_{34}$ & $X_{34,6}=0$ \\
\hline$X_{35}$ & $X_{35, i}=0(i=0, \ldots, 3,5,6,17, \ldots, 30)$ \\
\hline$X_{37}$ & $X_{37, i}=1(i=0,1,3, \ldots, 12)$ \\
\hline & $T_{1, i}=0(i=2,3,5), T_{2,17}=0, T_{3,7}=0, S_{1, i}=0(i=16,17), S_{2,16}=0$ \\
\hline
\end{tabular}


Table 11: The Extra Conditions of the Generalized Message Modification in the Right Branch

\begin{tabular}{|c|l|}
\hline $\begin{array}{c}\text { Chaining } \\
\text { Variable }\end{array}$ & Extra Conditions on the Chaining Variable \\
\hline$Y_{26}$ & $Y_{26, i}=0(i=29,30)$ \\
\hline$Y_{27}$ & $Y_{27, i}=0(i=3, \ldots, 6,13,14,22,25)$ \\
\hline$Y_{28}$ & $Y_{28, i}=0(i=3,6, \ldots, 9,23)$ \\
\hline & $\begin{array}{l}T_{4, i}=0(i=23,24), T_{5, i}=0(i=30,31), T_{7, i}=0(i=9,10) \\
\end{array}$ \\
$R_{2, i}=0(i=23,24,25), R_{5, i}=0(i=3,4,5), R_{9, i}=0(i=6,7,8)$ \\
\hline
\end{tabular}

